\title{
Consequences and Possible Predictors of Health-damaging Behaviors and Mental Health Problems in Pregnancy - A Review
}

\author{
Auswirkungen und mögliche Prädiktoren gesundheitsschädigender Verhaltensweisen und psychischer \\ Beeinträchtigungen in der Schwangerschaft - ein Überblick
}

Authors

Affiliation

\section{F. Ulrich, F. Petermann}

Zentrum für Klinische Psychologie und Rehabilitation der Universität Bremen, Bremen, Germany

\begin{abstract}
Key words
- early identification

- predictors

- risk factors

- pregnancy

- review

Schlüsselwörter

- Früherkennung

- Prädiktoren

- Risikofaktoren

- Schwangerschaft

- Übersicht
\end{abstract}

Deutsche Version unter: http://dx.doi.org/ 10.1055/s-0042-118180

received 23.6.2016

revised 13.9.2016

accepted 25.9.2016

\section{Bibliography}

DOI http://dx.doi.org/

10.1055/s-0042-118180

Geburtsh Frauenheilk 2016; 76:

1136-1156 @ Georg Thieme

Verlag KG Stuttgart · New York .

ISSN 0016-5751

\section{Correspondence}

\section{Dr. Franziska Ulrich,}

Dipl.-Psych.

Zentrum für Klinische

Psychologie und Rehabilitation

der Universität Bremen

Grazer Straße 6

28359 Bremen

Germany

f.ulrich@uni-bremen.de

\section{Abstract \\ $\nabla$}

In recent decades, the understanding of the short and longer term effects of health-damaging behaviors and mental health problems in pregnant women and the underlying mechanisms of these behaviors and illnesses has significantly increased. In contrast, little is known about the factors affecting individual pregnant women which contribute to health-damaging behaviors and mental illness. The aim of this paper was therefore to summarize the current state of research into the consequences of nicotine and alcohol consumption, malnutrition, excessive weight gain or obesity, and impaired mental health (depression and anxiety) during pregnancy. In addition, the characteristics of pregnant women which increase their risk of developing such behaviors or mental disorders are described. A better knowledge of these risks should make it easier for clinicians to identify cases at risk early on and put measures of support in place. A review of the literature has shown that certain characteristics of pregnant women (e.g. her relationship with her partner, a previous history of mental illness prior to pregnancy) are associated with various healthdamaging behaviors as well as with impaired mental health. Affected women often show an accumulated psychosocial stress which was already present prior to the pregnancy and which may persist even after the birth of the child.

\section{Introduction}

Because of their high developmental plasticity children are particularly sensitive to environmental factors in the early stages of their life $[1,2]$. Extensively investigated factors which can have last-

\section{Zusammenfassung \\ $\nabla$}

In den letzten Jahrzehnten hat sich das Wissen über die kurz- und langfristigen Effekte von gesundheitsschädigenden Verhaltensweisen und Beeinträchtigungen im psychischen Befinden der Schwangeren und deren zugrunde liegenden Mechanismen zunehmend verbessert. Weniger bekannt ist demgegenüber, welche Faktoren aufseiten der Schwangeren diese Risiken begünstigen können. Das Ziel dieses Beitrags ist es, den aktuellen Forschungsstand zu den Folgen eines Nikotinund Alkoholkonsums, einer Fehlernährung, einer starken Gewichtszunahme bzw. einer Adipositas sowie psychischer Beeinträchtigungen (Depression und Ängste) zusammenzufassen. Darüber hinaus werden Merkmale der schwangeren Frauen bestimmt, die das Auftreten dieser Risiken erhöhen können. Kenntnisse hierzu erleichtern es dem Kliniker, kritische Fälle frühzeitig zu identifizieren und bedarfsgerechte Unterstützungsangebote einzuleiten. Die Literaturdurchsicht hat gezeigt, dass bestimmte Merkmale der Schwangeren (z.B. Merkmale der Paarbeziehung, psychische Belastungen vor dem Einsetzen der Schwangerschaft) sowohl mit verschiedenen gesundheitsschädigenden Verhaltensweisen als auch mit Beeinträchtigungen im psychischen Befinden assoziiert sind. Die betroffenen Frauen weisen dabei zumeist eine Kumulation von psychosozialen Belastungen auf, die bereits vor der Schwangerschaft vorhanden sind, aber auch nach der Geburt des Kindes weiterhin bestehen bleiben können.

ing effects on pregnancy and birth and may result in long-term health impairments and mental disorders in children and adolescents include nicotine and alcohol consumption [3], nutritional deficiencies and malnutrition [4], excessive maternal weight gain or obesity during pregnancy [5], 
and impaired maternal mental health during pregnancy, for example as a result of high levels of stress or anxiety [6].

The association between early environmental influences and subsequent illnesses in children has justified the assumption of fetal or perinatal programming, whereby conditions in the womb such as suboptimal nourishment or increased concentrations of stress hormones at critical developmental stages trigger complex adaptation processes. This may result in permanent changes to the structure and functioning of various organs (e.g. the digestive, cardiovascular and central nervous system) $[7,8]$. This assumption is supported by an increased understanding of epigenetics which examines the molecular, immunological and endocrine mechanisms. These mechanisms are triggered by environmental factors (e.g. stress hormones) and lead to changes in gene activities but not to changes in the DNA sequence [9]. Maternal experiences and environmental factors in the perinatal period can increase or reduce gene expression, for example through DNA methylation or histone modification, thereby inducing longterm changes to various biological systems $[2,10]$. However, the understanding of the precise relationship between adverse intrauterine environmental factors and their potential impact on the physical or mental health of the child as well as the specific underlying mechanisms is still very limited and is far from fully elucidated. This is due to the fact that risk factors act in complicated ways, and that complex interrelations between genetic factors and environmental effects also have to be taken into account [7].

Despite these limitations, understanding the effects of intrauterine influences is of great practical relevance as it contributes to a better understanding of developmental psychopathological processes and shows how important it is to ensure that preventive measures are initiated as soon as possible [9]. The majority of health-damaging behaviors and psychological disorders are avoidable or can be changed by targeted preventive support and interventions in the perinatal period [1]. An understanding of the factors which promote health-damaging behaviors on the part of the pregnant woman or which increase her risk of developing a mental illness during pregnancy is particularly useful for early and effective detection and appropriate prevention measures and the promotion of better health. Previous research has focused on the impact of inequality due to social factors (e.g. low levels of education, low incomes) on physical and mental health [11-14]. However, what affects the pregnant woman's physical and psychological state of health is not so much her actual social status but instead the (socially determined) differences which manifest as specific burdens (e.g. housing conditions and conditions of employment) in the availability or lack of resources, in the prevalence of health-damaging behaviors, and in her medical care [11-12]. These factors, in their turn, can affect the capacity of the expectant mother to adjust to a variety of physiological, psychological and social changes during the perinatal period [15]. Previous reviews have devoted little attention to this aspect. This articles aims to start by summarizing previous findings on the consequences of health-damaging behaviors (nicotine and alcohol consumption, nutritional deficiencies or malnutrition, excessive weight gain and obesity) and psychological disorders for the pregnant woman and her unborn child and to provide an overview of the prevalence of these risks. In a second step, the results for possible factors which may promote health-damaging behaviors and mental health problems in pregnant women will be discussed. The paper focuses on those contributing factors which are linked primarily to the pregnant woman herself (e.g., age, stress, critical life events, pregnancy-specific factors) or to her immediate environment (e.g. aspects of her relationship with her intimate partner, the availability of social support). An extensive literature search was done using the Web of Science and PubMed. The following words or pairs of words were used as search terms:

- Pregnancy: ["pregnancy", "prenatal", "antenatal", "perinatal"];

- Nicotine and alcohol consumption: ["alcohol" OR "drinking" OR “smoking” OR "nicotine"];

- Nutrition and weight gain: ["nutrition" OR "malnutrition" OR "eating behavior", "obesity" OR "overweight" OR "weight gain"];

- Impaired mental health: ["depression" OR "anxiety" OR "mental health/illness" OR "stress"];

- Outcome: ["complications" OR "preterm delivery" OR "premature" OR "fetal growth", "birth weight" OR "behavior problems" OR "internalizing behavior" OR "externalizing behavior", "development"].

To determine influencing factors the words chosen as search terms were "correlates", "predictors" and "risks". The bibliographies of articles were also reviewed to find additional relevant articles.

\section{Selected Studies \\ $\nabla$}

Because of the abundance of studies, this review primarily looked at systematic reviews and meta-analyses. Individual studies were also included if, after reviewing their title and abstract, the studies met the following criteria:

- The study discussed either the impact of health-damaging behavior and/or mental health problems on the expectant mother (e.g. medical complications) or on her child.

- The study aimed to determine factors which promoted healthdamaging behaviors and/or mental disorders in the pregnant woman.

- Data was collected during pregnancy or at the time of giving birth at the latest.

- Only freely available publications in English or German were selected.

- The search was limited to journal articles published from January 2005 to July 2016 to ensure that all included studies were current.

- Studies which reported on infertility, multiple births, in vitro fertilization or surrogacy were excluded.

- Studies which analyzed the above-mentioned risk factors in the context of medical examinations (e.g. amniocentesis) or in high-risk groups (e.g. HIV infection) and studies which reported on the results of preventive programs and interventions were also disregarded.

\section{Nicotine and Alcohol Consumption \\ $\nabla$}

Nicotine and alcohol consumption are the most important modifiable risks for pregnant women and their unborn children [7, 16]. There is now a lot of evidence linking maternal nicotine consumption during pregnancy with permanent morphological changes (e.g. early termination of cell differentiation) and disorders of the neural transmitter systems in the fetal brain [17]. It is now also generally assumed that nicotine consumption impairs normal placental function and can result in oxygen deficiency 
(fetal hypoxia) and may reduce the supply of nutrition to the fetus [3]. Similar to nicotine, alcohol and its metabolites can pass across the placenta into the fetal blood stream and have a longterm negative effect on the organ development and brain maturation of the fetus [3].

\section{Effects of nicotine and alcohol consumption in pregnancy}

Medical complications affecting pregnant women as a result of nicotine consumption include premature rupture of membranes, placental abruption and placenta previa [18] as well as an increased risk of spontaneous abortion, stillbirth or premature delivery $[16,19,20]$. Intrauterine growth disorders [21], congenital anomalies [22], low birth weight, and the risk of sudden infant death syndrome [23] have also been linked to prenatal exposure to nicotine. Over the longer term maternal nicotine consumption during pregnancy is associated with an increased risk of health problems in later life, for example, cardiac and respiratory disease or overweight [16] but also with behavioral disorders (particularly ADHD and aggressive behavior) [24,25] and cognitive deficits [26] during the child's further development.

The harmful impact of chronic or high alcohol consumption as well as substance abuse and addiction during pregnancy on the unborn child have long been known [3]. The consequences for affected children can include prenatal and postnatal growth disorders, alcohol-related birth defects, developmental and behavioral disorders of varying severity, mental retardation and neuropsychological disorders [27]. Recent studies have increasingly focused on the impact of low to moderate alcohol consumption $(>0$ to 6 alcoholic drinks per week) and of episodic excessive alcohol consumption (so-called binge drinking; more than 4 to 5 alcoholic drinks per event). If women consume more than one or $1 \frac{1 / 2}{2}$ drinks containing alcohol per day during pregnancy, there is an increased risk of premature birth, intrauterine growth restriction and low birth weight [28]. A recent meta-analysis of 34 empirical studies published between 1988 and 2012 investigated the longterm effects of mild, moderate and binge-drinking prenatal exposure to alcohol on the neuropsychological functions of the affected children and the impact on the children's attention span, reading skills, executive function, and behavior [29]. The authors were able to show that even moderate quantities of alcohol were associated with adverse effects on the capacity to self-regulate behavior and on the capacity for interactive play, although the clinical relevance of these impairments were low to moderate. The cognitive functions of children aged between six months and 14 years were commonly found to be significantly adversely affected by maternal binge drinking during pregnancy [29]. Recent studies have made it clear that heavy episodic drinking on the part of the expectant mother in early and late pregnancy may be correlated with externalizing behaviour symptoms in affected children aged between five and seven years, even after controlling for additional variables (e.g. maternal level of education and maternal psychiatric disorder) $[30,31]$.

\section{Prevalence of nicotine and alcohol consumption in pregnancy}

Despite these adverse effects on the health of pregnant women and their unborn children, women continue to smoke and drink alcohol during pregnancy. In Germany there are only a few isolated recent prospective studies on the prevalence of nicotine and alcohol consumption by pregnant women. Depending on the time of the survey, the reported rates range from 11.2 to
$12.4 \%$ for maternal nicotine consumption during pregnancy [32, 33]. As far as we know, there are no recent prospective studies where the data was collected during pregnancy which investigated the prevalence of maternal alcohol consumption in Germany. The results of the German Health Interview and Examination Survey for Children and Adolescents (KiGGS study) were instructive, with the study showing that $13.5 \%$ of surveyed mothers reported having drunk alcohol during pregnancy [34].

\section{Correlates of nicotine and alcohol consumption in pregnancy}

The literature search showed that there are only two reviews which have looked at the different factors affecting maternal nicotine and/or alcohol consumption or abstention during pregnancy, one from 2010 and one from 2011 (cf. O Table 1). Another review focused entirely on the importance of social support during pregnancy on the pregnant woman's health-related behavior (particularly the impact of social support on nicotine and alcohol consumption). This prompted a search for other recent studies investigating factors which could be linked to continued nicotine and/or alcohol consumption. Overall, the results of a further 19 studies for the period from 2012 to 2015 were included in this review (cf. $\odot$ Table 2). The results for nicotine consumption and for alcohol consumption are reported below.

The pregnant woman's marital status and level of education were the most important socio-demographic and economic factors associated with maternal nicotine consumption during pregnancy (cf. $\odot$ Table 2). According to the data, single women with low levels of education were most likely to continue smoking during pregnancy $[32,35,51,56-58,61,65,67-68]$. Three studies found that women aged less than 25 years were the most likely to continue smoking despite their pregnancy $[32,61,62]$, while the findings of Kharkova et al. [57] showed the opposite. Social integration and the availability of emotional support, and of informal and practical help were important resources for successfully managing stressful events and had a correspondingly positive impact on the physical and mental health [88]. Social support also played an important role in helping pregnant women stop smoking [89]. Pregnant women who continued to smoke were more likely to experience conflicts with their partner $[54,59]$ and were less likely to feel they were supported by their partner or by close relatives or friends or were less satisfied with the perceived support they received from their partner [37]. It was particularly difficult for the pregnant woman to stop smoking if her partner smoked [35].

In addition to social characteristics there was repeated evidence for the existence of mental health problems or stress among pregnant women who smoked, usually in the form of increased depression and anxiety scores $[51,53,54,59,68]$. Women who continued to smoke during pregnancy were almost twice as likely to be depressed compared to women who had stopped smoking [68]. Critical life events and high levels of perceived stress could also make it more difficult to quit smoking during pregnancy [54]. Pregnant immigrant women appeared to smoke less than non-immigrant women [32]; psychological problems were a major factor associated with prenatal nicotine consumption by immigrant women [61]

Women's smoking behavior, the amount of nicotine consumed and frequency with which it was consumed prior to becoming pregnant were not unimportant health-related factors [35]. Women who smoked more than 10 cigarettes daily before pregnancy found it much more difficult to quit or reduce their smok- 
Table 1 Findings of reviews and meta-analyses on factors affecting the health and behavioral risk-taking by pregnant women.

\begin{tabular}{|c|c|c|c|c|}
\hline Authors & $\begin{array}{l}\text { Maternal } \\
\text { risk factor }\end{array}$ & Influencing factors & $\begin{array}{l}\text { Information about } \\
\text { the study }\end{array}$ & Main findings and comments \\
\hline \multicolumn{5}{|c|}{ Nicotine and alcohol consumption } \\
\hline$[35]^{\mathrm{a}}$ & $\begin{array}{l}\text { Nicotine } \\
\text { consumption }\end{array}$ & $\begin{array}{l}\text { Social factors, nicotine } \\
\text { consumption behavior, } \\
\text { social relationships, } \\
\text { pregnancy-specific } \\
\text { factors }\end{array}$ & $\begin{array}{l}n=19 \text { studies } \\
\text { from } 1997 \text { to } 2008\end{array}$ & $\begin{array}{l}\text { Significant correlation between prenatal nicotine consumption } \\
\text { and the following factors: } \\
\text { income ( } 4 \text { of } 5 \text { studies); partner who smokes ( } 6 \text { of } 6 \text { studies); high number } \\
\text { of children ( } 11 \text { of } 12 \text { studies); high nicotine consumption ( } 7 \text { of } 8 \text { studies); } \\
\text { prenatal care appointments attended less often and only during the later } \\
\text { stages of pregnancy ( } 4 \text { of } 5 \text { studies). }\end{array}$ \\
\hline$[36]^{a}$ & $\begin{array}{l}\text { Alcohol } \\
\text { consumption }\end{array}$ & $\begin{array}{l}\text { Psychosocial and } \\
\text { behavioral factors }\end{array}$ & $\begin{array}{l}n=14 \text { studies } \\
\text { ( } \mathrm{n}=11 \text { cross-sectional } \\
\text { studies; } \mathrm{n}=3 \\
\text { longitudinal studies) } \\
\text { from } 2002 \text { to } 2009\end{array}$ & $\begin{array}{l}\text { Significant and consistent correlations between prenatal alcohol } \\
\text { consumption and pre-pregnancy alcohol consumption ( } 7 \text { of } 7 \text { studies) } \\
\text { and experience of abuse/violence ( } 3 \text { of } 3 \text { studies). Significant correlation } \\
\text { with higher age ( } 7 \text { of } 12 \text { studies), nicotine consumption ( } 5 \text { of } 10 \text { studies) } \\
\text { and larger number of children ( } 4 \text { of } 10 \text { studies). } \\
\text { Inconsistent findings with regard to socio-economic status, level } \\
\text { of education, unemployment, marital status and alcohol dependency. }\end{array}$ \\
\hline$[37]^{\mathrm{a}}$ & $\begin{array}{l}\text { Nicotine and } \\
\text { alcohol } \\
\text { consumption }\end{array}$ & Social support & $\begin{array}{l}\mathrm{n}=13 \text { studies } \\
2003-2013\end{array}$ & $\begin{array}{l}\text { Significant correlations between prenatal nicotine consumption } \\
\text { and social support ( } 7 \text { of } 10 \text { studies); no consistent correlation with } \\
\text { prenatal alcohol consumption ( } 1 \text { of } 3 \text { studies). }\end{array}$ \\
\hline \multicolumn{5}{|c|}{ Diet and weight gain/obesity } \\
\hline$[37]^{\mathrm{a}}$ & Nutrition & Social support & $\begin{array}{l}\mathrm{n}=3 \text { studies } \\
2003-2013\end{array}$ & $\begin{array}{l}\text { Significant correlations between social support and satisfaction } \\
\text { with social support and nutrition for women on low incomes and } \\
\text { with an immigrant background ( } 2 \text { of } 3 \text { studies). } \\
\text { No meaningful results possible because of the limited number of studies. }\end{array}$ \\
\hline$[38]^{\mathrm{a}}$ & $\begin{array}{l}\text { Quality } \\
\text { of food }\end{array}$ & $\begin{array}{l}\text { Mental health } \\
\text { of the mother in the } \\
\text { perinatal period }\end{array}$ & $\begin{array}{l}n=9 \text { studies } \\
(n=4 \text { cohort studies; } \\
n=5 \text { cross-sectional } \\
\text { studies) } \\
2005-2013\end{array}$ & $\begin{array}{l}\text { Significant correlations between unhealthy diet and poor quality food } \\
\text { and prenatal depressive symptoms ( } 3 \text { of } 3 \text { studies) and stress symptoms } \\
\text { ( } 3 \text { of } 3 \text { studies). } \\
\text { Only limited significance because of the low number of studies. } \\
\text { No statements can be made on the impact of factors. } \\
\text { Other factors such as social support, mental illness prior to pregnancy } \\
\text { and socio-economic factors could be important. }\end{array}$ \\
\hline$[39]^{a}$ & $\begin{array}{l}\text { Excessive } \\
\text { weight gain }\end{array}$ & $\begin{array}{l}\text { Anxiety, depression, } \\
\text { stress, social support, } \\
\text { dissatisfied with body } \\
\text { image, self-esteem } \\
\text { and self-efficacy }\end{array}$ & $\begin{array}{l}\mathrm{n}=12 \text { studies } \\
\text { ( } \mathrm{n}=2 \text { cross-sectional } \\
\text { studies; } \mathrm{n}=8 \\
\text { longitudinal studies; } \\
\mathrm{n}=2 \text { randomized } \\
\text { control group studies) } \\
2000-2014\end{array}$ & $\begin{array}{l}\text { Significant correlations between excessive weight gain and depression } \\
\text { ( } 2 \text { of } 2 \text { studies); dissatisfaction with own body image ( } 4 \text { of } 6 \text { studies) } \\
\text { and social support ( } 1 \text { study). } \\
\text { No statements possible on the impact of factors. }\end{array}$ \\
\hline$[40]^{\mathrm{a}}$ & $\begin{array}{l}\text { Excessive } \\
\text { weight gain }\end{array}$ & $\begin{array}{l}\text { Psychological charac- } \\
\text { teristics (affective } \\
\text { state, cognition, } \\
\text { motive for weight gain } \\
\text { and diet) }\end{array}$ & $\begin{array}{l}\mathrm{n}=35 \text { studies } \\
\text { ( } \mathrm{n}=8 \text { cross-sectional } \\
\text { studies; } \mathrm{n}=25 \text { cohort } \\
\text { studies; } \mathrm{n}=2 \text { case } \\
\text { control studies) }\end{array}$ & $\begin{array}{l}\text { Significant correlations between excessive weight gain and weight-related } \\
\text { and diet-related cognition: } \\
\text { dissatisfaction with own body image ( } 2 \text { of } 4 \text { studies); negative attitude } \\
\text { to weight gain ( } 4 \text { of } 6 \text { studies); inaccurate perceptions of own weight both } \\
\text { in women with normal weight and in overweight women ( } 1 \text { of } 2 \text { studies); } \\
\text { higher levels of cognitive dietary restraint ( } 2 \text { of } 3 \text { studies). } \\
\text { Additional predictors which will need further study include perceived } \\
\text { barriers to healthy eating ( } 1 \text { study); concern about weight gain ( } 1 \text { study); } \\
\text { limited knowledge about weight gain ( } 1 \text { study). } \\
\text { Negative affective states (anxiety, depression and stress) were not found } \\
\text { to be related to weight gain. }\end{array}$ \\
\hline$[41]^{a, b}$ & $\begin{array}{l}\text { Obesity and } \\
\text { overweight }\end{array}$ & $\begin{array}{l}\text { Mental health prob- } \\
\text { lems during pregnancy } \\
\text { and post partum }\end{array}$ & $\mathrm{n}=62$ studies & $\begin{array}{l}\text { Obese/overweight pregnant women had an increased risk of prenatal } \\
\text { depression (obese pregnant women } O R=1.43 \text {; overweight pregnant } \\
\text { women } O R=1.19 \text { ); of postpartum depression (obese pregnant women: } \\
\mathrm{OR}=1.30 \text {; overweight pregnant women: } \mathrm{OR}=1.09 \text { ), and an increased risk } \\
\text { of anxiety symptoms during pregnancy }(\mathrm{OR}=1.41 \text { ). }\end{array}$ \\
\hline$[42]^{\mathrm{a}}$ & $\begin{array}{l}\text { Excessive } \\
\text { weight gain }\end{array}$ & $\begin{array}{l}\text { Maternal symptoms } \\
\text { of anxiety }\end{array}$ & $\begin{array}{l}n=13 \text { studies } \\
\text { ( } n=10 \text { longitudinal } \\
\text { studies; } n=3 \text { inter- } \\
\text { ventional studies) } \\
\text { from } 2000 \text { to } 2015\end{array}$ & $\begin{array}{l}\text { Significant correlation between obesity and anxiety experienced by } \\
\text { the expectant mother ( } 5 \text { of } 7 \text { studies). } \\
\text { Results should be viewed as a comorbidity as anxiety symptoms } \\
\text { were not recorded prior to the pregnancy. }\end{array}$ \\
\hline \multicolumn{5}{|c|}{ Mental health problems } \\
\hline$[43]^{\mathrm{a}, \mathrm{b}}$ & $\begin{array}{l}\text { Perinatal } \\
\text { depression }\end{array}$ & Unwanted pregnancy & $\begin{array}{l}\mathrm{n}=10 \text { studies } \\
\text { ( } \mathrm{n}=5 \text { cross-sectional } \\
\text { studies; } \mathrm{n}=4 \\
\text { longitudinal studies; } \\
\mathrm{n}=1 \text { randomized } \\
\text { control group study) } \\
\text { from } 1991 \text { to } 2014\end{array}$ & $\begin{array}{l}\text { Prevalence of maternal perinatal depression associated with unwanted } \\
\text { pregnancy: } 21 \% \\
\text { Note: Only } 3 \text { studies collected data at the time of the pregnancy itself. } \\
\text { Different instruments were used to record or measure depression. }\end{array}$ \\
\hline
\end{tabular}


Table 1 Findings of reviews and meta-analyses on factors affecting the health and behavioral risk-taking by pregnant women. (Continued)

\begin{tabular}{|c|c|c|c|c|}
\hline Authors & $\begin{array}{l}\text { Maternal } \\
\text { risk factor }\end{array}$ & Influencing factors & $\begin{array}{l}\text { Information about } \\
\text { the study }\end{array}$ & Main findings and comments \\
\hline$[44]^{\mathrm{a}}$ & $\begin{array}{l}\text { Perinatal } \\
\text { depression }\end{array}$ & Experience of abuse & $\begin{array}{l}n=43 \text { studies } \\
\text { ( } \mathrm{n}=29 \text { cross-sectional } \\
\text { studies; } \mathrm{n}=14 \\
\text { longitudinal studies) } \\
\text { to } 2011\end{array}$ & $\begin{array}{l}\text { Every form of abuse (direct, indirect, physical, sexual or emotional abuse) } \\
\text { was found to be positively correlated with prenatal and postnatal } \\
\text { depression (only } 5 \text { studies found no significant association). }\end{array}$ \\
\hline$[45]^{a}$ & $\begin{array}{l}\text { Prenatal } \\
\text { anxiety and } \\
\text { depression }\end{array}$ & $\begin{array}{l}\text { Psychosocial, } \\
\text { gynecological and } \\
\text { behavioral factors }\end{array}$ & $\begin{array}{l}\mathrm{n}=97 \text { studies } \\
\text { from } 2003 \text { to } 2015\end{array}$ & $\begin{array}{l}\text { Significant association between prenatal depression/anxiety } \\
\text { and the following factors: } \\
\text { lack of support by intimate partner or lack of social support (sign. predictor } \\
\text { in } 29 \text { studies vs. not a sign. predictor in } 0 \text { studies); experience of previous } \\
\text { abuse or violence by partner (sign. predictor in } 28 \text { studies vs. not a sign. } \\
\text { predictor in } 0 \text { studies); adverse life events and high levels of perceived stress } \\
\text { (sign. predictor in } 21 \text { studies vs. not a sign. predictor in } 0 \text { studies); previous } \\
\text { mental illness (sign. predictor in } 23 \text { studies vs. not a sign. predictor in } \\
0 \text { studies); unplanned or unwanted pregnancy (sign. predictor in } 22 \text { studies } \\
\text { vs. not a sign. predictor in } 2 \text { studies); current/previous pregnancy } \\
\text { complications/pregnancy loss (sign. predictor in } 17 \text { studies vs. not } \\
\text { a sign. predictor in } 4 \text { studies); nicotine consumption during pregnancy } \\
\text { (sign. predictor in } 11 \text { studies vs. not a sign. predictor in } 1 \text { study). } \\
\text { More research is needed with regard to the following risk factors: } \\
\text { alcohol abuse; substance abuse; familial history of mental illness; } \\
\text { partner unemployed; quality of upbringing; mode of delivery. }\end{array}$ \\
\hline$[46]^{a}$ & $\begin{array}{l}\text { Perinatal } \\
\text { psychological } \\
\text { disorders }\end{array}$ & $\begin{array}{l}\text { Psychosocial and } \\
\text { gynecological factors }\end{array}$ & $\begin{array}{l}n=47 \text { studies } \\
\text { ( } \mathrm{n}=13 \text { studies where } \\
\text { data was collected } \\
\text { during pregnancy; } \\
\mathrm{n}=34 \text { studies where } \\
\text { data was collected } \\
\text { after the birth) } \\
\text { to } 2010\end{array}$ & $\begin{array}{l}\text { Significant associations between perinatal mental disorders } \\
\text { (mainly anxiety/depression) and the following factors: } \\
\text { socio-economic disadvantage }(O R=2.1-13.2) \text {; unintended pregnancy } \\
(\mathrm{OR}=1.6-8.8) \text {; young maternal age }(\mathrm{OR}=2.1-5.4) \text {; unmarried }(\mathrm{OR}=3.4- \\
5.8) \text {; lack of intimate partner empathy or support }(\mathrm{OR}=2.0-9.4) \text {; hostile } \\
\text { in-laws }(\mathrm{OR}=2.1-4.4) \text {; experienced intimate partner violence }(\mathrm{OR}=2.11 \text { - } \\
6.75) \text {; lack of practical and emotional support }(\mathrm{OR}=2.8-6.1) \text {; child is female } \\
(\mathrm{OR}=1.8-2.6) \text {; previous maternal history of mental illness }(\mathrm{OR}=5.1-5.6) \text {. }\end{array}$ \\
\hline$[47]^{a, b}$ & $\begin{array}{l}\text { Perinatal } \\
\text { psychological } \\
\text { disorders }\end{array}$ & Domestic violence & $\begin{array}{l}n=67 \text { studies } \\
\text { ( } \mathrm{n}=51 \text { cross-sectional } \\
\text { studies; } \mathrm{n}=16 \\
\text { longitudinal studies) } \\
\text { up to } 2011\end{array}$ & $\begin{array}{l}\text { Women with prenatal or postpartum depression had a three to five times } \\
\text { higher risk of experiencing violence: } \\
\text { experience of violence for women with prenatal depression (experienced } \\
\text { violence over the patient's lifetime: } \mathrm{OR}=3.0 \text {, during the previous year: } \\
\mathrm{OR}=2.8 \text {, or during the pregnancy: } \mathrm{OR}=5.0 \text { ); Violence experienced by } \\
\text { women with postpartum depression: (experienced violence during the } \\
\text { previous year: } \mathrm{OR}=2.9 \text {; or during the pregnancy: } \mathrm{OR}=3.1 \text { ). } \\
\text { Women with prenatal symptoms of anxiety had an up to } 2.9 \text {-times higher } \\
\text { risk of experiencing violence. } \\
\text { Women with prenatal or postnatal PTSD had a } 4.6 \text { - to } 6.4 \text {-times higher risk } \\
\text { of experiencing violence. }\end{array}$ \\
\hline$[48]^{\mathrm{a}}$ & $\begin{array}{l}\text { Prenatal } \\
\text { depression }\end{array}$ & $\begin{array}{l}\text { Psychosocial, } \\
\text { gynecological and } \\
\text { behavioral factors }\end{array}$ & $\begin{array}{l}n=57 \text { studies } \\
\text { from } 1980 \text { to } 2008\end{array}$ & $\begin{array}{l}\text { Significant correlations between prenatal depressive symptoms } \\
\text { and the following factors: } \\
\text { stressful life events; lack of social support, and domestic violence. }\end{array}$ \\
\hline [49] & $\begin{array}{l}\text { Perinatal } \\
\text { anxiety } \\
\text { disorders }\end{array}$ & $\begin{array}{l}\text { Psychosocial, } \\
\text { gynecological and } \\
\text { behavioral factors }\end{array}$ & $\begin{array}{l}n=98(n=47 \text { studies } \\
\text { on predictors) } \\
\text { from } 2006 \text { to } 2014\end{array}$ & $\begin{array}{l}\text { Young age ( } 5 \text { studies); single/no partner ( } 6 \text { studies); low socio-economic } \\
\text { status ( } 5 \text { studies), low level of education ( } 9 \text { studies); lack of social support } \\
\text { ( } 7 \text { studies); conflicts in relationship with partner ( } 5 \text { studies), previous } \\
\text { mental illness ( } 3 \text { studies); obstetrical complications: previous miscarriage/ } \\
\text { spontaneous abortion ( } 5 \text { studies); unplanned pregnancy; poor health/ } \\
\text { pregnancy complications ( } 3 \text { studies). }\end{array}$ \\
\hline$[50]^{a, b}$ & $\begin{array}{l}\text { Perinatal } \\
\text { depression } \\
\text { and anxiety }\end{array}$ & $\begin{array}{l}\text { Risk factors and } \\
\text { protective factors } \\
\text { affected by partner }\end{array}$ & $\begin{array}{l}n=120 \text { studies } \\
\text { ( } 62 \% \text { cross-sectional } \\
\text { studies; } 37 \% \\
\text { longitudinal studies) } \\
\text { from } 1980 \text { to } 2013\end{array}$ & $\begin{array}{l}\text { Partner factors associated with a risk of prenatal depression or anxiety: } \\
\text { conflicts (depression } r=0.30 \text {; anxiety } r=0.35 \text { ); partner consumes alcohol } \\
\text { and drugs (for depression } r=0.16 \text { ); emotional withdrawal of partner } \\
\text { (for depression } r=0.32 \text { ). } \\
\text { Partner factors which reduce the risk of prenatal depression or anxiety: } \\
\text { emotional closeness (for depression } r=-0.25 \text {; for anxiety } r=-0.22 \text { ); } \\
\text { emotional support (for depression } r=-0.26 \text {; for anxiety } r=-0.20 \text { ), } \\
\text { and global support (for depression } r=-0.28 \text {; for anxiety } r=-0.32 \text { ). } \\
\text { Additional factors specifically associated with prenatal depression: } \\
\text { positive communication ( } r=-0.31 \text { ); instrumental support }(r=-0.16 \text { ), } \\
\text { and relationship satisfaction }(r=-0.29 \text { ). }\end{array}$ \\
\hline
\end{tabular}

OR: odds ratio. PTSD: post-traumatic stress disorder. r = correlation coefficient. sign.: significant.

a: Systematic review.

b: Meta-analysis. 
Table 2 Findings of empirical studies on factors influencing health-damaging behaviors and mental health problems by pregnant women.

\begin{tabular}{|c|c|c|c|c|c|c|}
\hline Authors & Country & Sample & Study design & $\begin{array}{l}\text { Time of data } \\
\text { collection }\end{array}$ & Prevalence & Factors which influenced behaviors \\
\hline \multicolumn{7}{|c|}{ Nicotine and alcohol consumption } \\
\hline [32] & Germany & $\begin{array}{l}\mathrm{n}=647392 \text { pregnant } \\
\text { women }\end{array}$ & $\begin{array}{l}\text { Population- } \\
\text { based cross- } \\
\text { sectional } \\
\text { study }\end{array}$ & $\begin{array}{l}\text { Information } \\
\text { obtained from } \\
\text { maternity log } \\
2005\end{array}$ & $\begin{array}{l}12.4 \% \text { of pregnant } \\
\text { women smoked } \\
(M=10 \text { cigarettes } \\
\text { per day). }\end{array}$ & $\begin{array}{l}\text { Factors associated with continued consump- } \\
\text { tion of nicotine: socio-economic status } \\
\text { (housewife: } \mathrm{OR}=3.09 \text {; unskilled worker: } \\
\mathrm{OR}=4.44 \text { ); age }(<20 \text { years: } \mathrm{OR}=5.96) \text {; } \text { num- } \\
\text { ber of previous births (more than } 2 \text { children: } \\
\mathrm{OR}=2.64 \text { ); inadequate prenatal care } \\
\text { (fewer than } 5 \text { antenatal care appointments: } \\
\mathrm{OR}=1.76 \text { ) }\end{array}$ \\
\hline [51] & $\begin{array}{l}\text { Nether- } \\
\text { lands }\end{array}$ & $\begin{array}{l}n=6104 \text { pregnant } \\
\text { women } \\
(M=30.4 \text { years; } \\
S D=4.6)\end{array}$ & $\begin{array}{l}\text { Prospective } \\
\text { population- } \\
\text { based cohort } \\
\text { study } \\
\text { (DELIVER } \\
\text { study) }\end{array}$ & $\begin{array}{l}\text { 34th GW } \\
\text { (median } \\
=19 \text { th GW) } \\
\text { 2009-2011 }\end{array}$ & $\begin{array}{l}9.2 \% \text { of pregnant } \\
\text { women continued } \\
\text { smoking. } \\
\text { Maternal smokers } \\
\text { smoked an average } \\
\text { of } 7.8 \text { cigarettes per } \\
\text { day }(S D=4.4 \text {, range } \\
1.5-20) \text {; occasional } \\
\text { smokers consumed } \\
8.8 \text { cigarettes } \\
\text { per week }(S D=8.1 \text {, } \\
\text { range } 0-50) \text {. }\end{array}$ & $\begin{array}{l}\text { Predictors for any amount of nicotine } \\
\text { consumption: } \\
\text { low level of education ( } O R=10.3) \text {; Turkish } \\
\text { ethnicity }(O R=3.9) \text {; no partner }(O R=3.7) \text {; } \\
\text { unplanned pregnancy }(O R=1.4) \text {; under- } \\
\text { weight }(O R=2.1) \text {; alcohol consumed during } \\
\text { pregnancy }(O R=1.4) \text {; low health-related lo- } \\
\text { cus of control }(O R=1.4) \text {; increased anxiety } \\
\text { and depression scores }(O R=1.8) \text {. } \\
\text { Women who smoked every day were more } \\
\text { likely to have an unplanned pregnancy } \\
(O R=1.5) \text {, be underweight }(O R=2.6) \text { and } \\
\text { not take folic acid supplements }(O R=1.6) \text {. }\end{array}$ \\
\hline [52] & $\begin{array}{l}\text { Nether- } \\
\text { lands }\end{array}$ & $\begin{array}{l}n=2287 \text { pregnant } \\
\text { women ( } n=113 \\
\text { smokers; } M=30.5 \\
\text { years; } S D=5.6) ; \\
(n=290 \text { former } \\
\text { smokers; } M=30.6 \\
\text { years; } S D=4.9) ; \\
(n=1863 \text { non-smok- } \\
\text { ers; } M=31.8 \text { years; } \\
S D=4.4) ;(n=124 \\
\text { women who con- } \\
\text { tinued to consume } \\
\text { alcohol; } M=32.8 \\
\text { years; } S D=4.4 \text { ); } \\
\text { ( } n=1403 \text { women } \\
\text { who stopped drink- } \\
\text { ing alcohol; } M=31.7 \\
\text { years; } S D=4.5 \text { ); } \\
(n=760 \text { teetotalers; } \\
M=31.2 \text { years; } \\
S D=4.7 \text { ) }\end{array}$ & $\begin{array}{l}\text { Prospective } \\
\text { population- } \\
\text { based cohort } \\
\text { study (PAD) }\end{array}$ & $\begin{array}{l}\text { 19th GW } \\
\text { 2011-2013 }\end{array}$ & $\begin{array}{l}28 \% \text { of pregnant } \\
\text { women continued } \\
\text { smoking; } 8.1 \% \text { of } \\
\text { pregnant women } \\
\text { continued to } \\
\text { consume alcohol. }\end{array}$ & $\begin{array}{l}\text { Continued alcohol consumption was asso- } \\
\text { ciated with the following factors: } \\
\text { Conflict with loved ones }(\mathrm{OR}=10.4) \text {; crime- } \\
\text { related events }(\mathrm{OR}=35.7) \text {; pregnancy-specif- } \\
\text { ic events ( } O R=13.4) \text {, and number of stressful } \\
\text { events ( } O R=17.2) \text {. } \\
\text { No association was found with anxiety/ } \\
\text { depression. }\end{array}$ \\
\hline [53] & Belgium & $\begin{array}{l}n=523 \text { pregnant } \\
\text { women }(M=29 \\
\text { years; } S D=4.38)\end{array}$ & $\begin{array}{l}\text { Prospective } \\
\text { longitudinal } \\
\text { study }\end{array}$ & $\begin{array}{l}\mathrm{t}_{0}: 16 \text { th } \mathrm{GW} \\
\mathrm{t}_{1}: 32 \mathrm{nd} \text { to } \\
34 \text { th GW } \\
\mathrm{t}_{2}: 96 \text { weeks } \\
\text { postpartum } \\
2008-2010\end{array}$ & $\begin{array}{l}16.3 \% \text { of pregnant } \\
\text { women smoked. }\end{array}$ & $\begin{array}{l}\text { Smokers reported significantly more depres- } \\
\text { sive symptoms at all three time-points of } \\
\text { data collection compared to non-smokers } \\
\text { and women who had stopped smoking. } \\
\text { The differences were particularly apparent } \\
\text { among women with low levels of education. }\end{array}$ \\
\hline [54] & Norway & $\begin{array}{l}\mathrm{n}=73418 \text { pregnant } \\
\text { women (no data } \\
\text { given regarding age) }\end{array}$ & $\begin{array}{l}\text { Prospective } \\
\text { population- } \\
\text { based cohort } \\
\text { study (MoBa) }\end{array}$ & $\begin{array}{l}\mathrm{t}_{1}: 17 \text { th and } \\
30 \text { th GW } \\
\mathrm{t}_{2}: 6 \text { months } \\
\text { postpartum } \\
1999-2008\end{array}$ & $\begin{array}{l}27.5 \% \text { of women } \\
\text { smoked prior to } \\
\text { pregnancy; } 44.2 \% \\
\text { of them continued } \\
\text { smoking. }\end{array}$ & $\begin{array}{l}\text { Women with high anxiety and depression } \\
\text { scores were less likely to quit smoking during } \\
\text { pregnancy }(\mathrm{OR}=0.80) \text { and more likely to } \\
\text { smoke before becoming pregnant } \\
(\mathrm{OR}=1.45) \text {. Relationship conflicts ( } \mathrm{OR}=0.82) \\
\text { and negative life events ( } \mathrm{OR}=0.93 \text { ) also had a } \\
\text { negative effect on the likelihood of quitting } \\
\text { smoking. }\end{array}$ \\
\hline [55] & $\begin{array}{l}\text { Den- } \\
\text { mark }\end{array}$ & $\begin{array}{l}n=3238 \text { pregnant } \\
\text { women }(M=32 \\
\text { years; } S D=5)\end{array}$ & $\begin{array}{l}\text { Prospective } \\
\text { population- } \\
\text { based cohort } \\
\text { study (Copen- } \\
\text { hagen Preg- } \\
\text { nancy Cohort } \\
\text { Study) }\end{array}$ & $\begin{array}{l}\text { 10th GW } \\
\text { 2012-2013 }\end{array}$ & $\begin{array}{l}3 \% \text { of women drank } \\
\text { alcohol every week } \\
\text { during early preg- } \\
\text { nancy; } 35 \% \text { of them } \\
\text { reported binge } \\
\text { drinking in early } \\
\text { pregnancy. }\end{array}$ & $\begin{array}{l}\text { Factors which facilitated binge drinking: } \\
\text { Alcohol consumption prior to pregnancy } \\
\text { (one glass per week: } \mathrm{aOR}=4.48 ; 2-7 \text { glasses } \\
\text { per week: } \mathrm{aOR}=10.23 ; \geq 8 \text { glasses per week: } \\
\text { aOR=33.18); nicotine consumption prior } \\
\text { to pregnancy (aOR=2.24), and unplanned } \\
\text { pregnancy ( } \mathrm{aOR}=2.74 \text { ). }\end{array}$ \\
\hline
\end{tabular}


Table 2 Findings of empirical studies on factors influencing health-damaging behaviors and mental health problems by pregnant women. (Continued)

\begin{tabular}{|c|c|c|c|c|c|c|}
\hline Authors & Country & Sample & Study design & $\begin{array}{l}\text { Time of data } \\
\text { collection }\end{array}$ & Prevalence & Factors which influenced behaviors \\
\hline [56] & Brazil & $\begin{array}{l}n=1744 \text { pregnant } \\
\text { women ( } n=549 \text { from } \\
\text { Niterói; } 60.8 \% \text { aged } \\
20-34 \text { years); } \\
\text { ( } n=1195 \text { from } \\
\text { Rio de Janeiro; } 65.4 \% \\
\text { aged } 20-34 \text { years) }\end{array}$ & $\begin{array}{l}\text { Cross- } \\
\text { sectional } \\
\text { study } \\
\text { data from } \\
2011\end{array}$ & $\begin{array}{l}12 \text { hours after } \\
\text { birth } \\
2011\end{array}$ & $\begin{array}{l}17.9-24.8 \% \text { of } \\
\text { women smoked } \\
\text { during pregnancy. }\end{array}$ & $\begin{array}{l}\text { Factors associated with continued nicotine } \\
\text { consumption: } \\
\text { low level of education ( } O R=2.14 \text { Niterói; } \\
O R=1.61 \text { Rio de Janeiro); multiparity } \\
(O R=3.48 \text { Niterói; } O R=1.58 \text { Rio de Janeiro). }\end{array}$ \\
\hline [57] & $\begin{array}{l}\text { Russia } \\
\text { (Mur- } \\
\text { mansk) }\end{array}$ & $\begin{array}{l}\mathrm{n}=12871 \text { pregnant } \\
\text { women, } 34.9 \% \text { aged } \\
20-24 \text { years, } 29.4 \% \\
\text { aged } 25-29 \text { years, } \\
16.6 \% \text { aged } \\
30-34 \text { years })\end{array}$ & $\begin{array}{l}\text { Population- } \\
\text { based cross- } \\
\text { sectional } \\
\text { study }\end{array}$ & $\begin{array}{l}\text { Survey carried } \\
\text { out while } \\
\text { women were } \\
\text { in the mater- } \\
\text { nity hospital } \\
\text { 2006-2011 }\end{array}$ & $\begin{array}{l}25.2 \% \text { women } \\
\text { smoked prior to } \\
\text { becoming pregnant } \\
\text { and } 18.9 \% \text { of } \\
\text { women smoked } \\
\text { during pregnancy. }\end{array}$ & $\begin{array}{l}\text { Factors which facilitated the cessation } \\
\text { of smoking: } \\
\text { Younger women ( } \leq 20-24 \text { years: } O R=1.19 \text { ) } \\
\text { were more likely to reduce their nicotine con- } \\
\text { sumption than older women ( } 30-34 \text { years: } \\
\text { OR }=0.98 ; \geq 35 \text { years: } O R=0.82 \text { ); nullipara } \\
\text { and secundigravida were more likely to re- } \\
\text { duce their nicotine consumption than multi- } \\
\text { para (nullipara: } O R=2.21 \text { or already one child: } \\
\text { OR= } 1.69 \text { ). } \\
\text { Factors which made it less likely that the } \\
\text { pregnant woman would reduce or quit } \\
\text { smoking: } \\
\text { low level of education (OR }=0.39 \text { ); marital } \\
\text { status (single: } O R=0.53 \text { or with partner: } \\
\text { OR=0.49); place of residence } \\
\text { (rural area: } O R=0.76 \text { ), and ethnicity } \\
\text { (non-Russian ethnicity: OR=0.90). }\end{array}$ \\
\hline [58] & USA & $\begin{array}{l}n=902 \text { pregnant } \\
\text { women }(M=24.8 \\
\text { years; } S D=5.9)\end{array}$ & $\begin{array}{l}\text { Cross- } \\
\text { sectional } \\
\text { study }\end{array}$ & $\begin{array}{l}\text { 1st prenatal } \\
\text { care appoint- } \\
\text { ment } \\
(M=15.1 \mathrm{GW} ; \\
\mathrm{SD}=8.3) \\
2009-2011\end{array}$ & $\begin{array}{l}17 \% \text { of pregnant } \\
\text { women smoked } \\
\text { at the time of the } \\
\text { prenatal care } \\
\text { appointment. }\end{array}$ & $\begin{array}{l}\text { Factors associated with continued nicotine } \\
\text { consumption: } \\
\text { maternal age }(O R=1.08) \text {; low level of educa- } \\
\text { tion }(O R=4.30) \text {; unemployed ( } O R=2.33) \text {; } \\
\text { criminal history }(O R=1.66) \text {; in receipt of social } \\
\text { services }(O R=2.26) \text {; alcohol consumption } \\
(O R=2.73) \text {, and consumption of illegal drugs } \\
(O R=1.97) \text { during pregnancy. }\end{array}$ \\
\hline [59] & USA & $\begin{array}{l}n=1518 \text { pregnant } \\
\text { women }(73.1 \% \text { aged } \\
20-34 \text { years })\end{array}$ & $\begin{array}{l}\text { Cross- } \\
\text { sectional } \\
\text { study }\end{array}$ & $\begin{array}{l}\text { Between 18th } \\
\text { and 28nd GW }\end{array}$ & $\begin{array}{l}17.6 \% \text { of women } \\
\text { smoked during } \\
\text { pregnancy. }\end{array}$ & $\begin{array}{l}\text { Factors associated with continued nicotine } \\
\text { consumption: } \\
\text { Higher levels of perceived stress ( } O R=1.76) \text {; } \\
\text { depression }(O R=1.48) \text {; neuroticism } \\
(\mathrm{OR}=1.58) \text {; negative behavior by partner } \\
\text { (control, criticism, abuse) }(\mathrm{OR}=1.15) \text {, and } \\
\text { perceived racism }(\mathrm{OR}=1.15) \text { were associated } \\
\text { with nicotine consumption. }\end{array}$ \\
\hline [60] & Canada & $\begin{array}{l}\mathrm{n}=2246 \text { pregnant } \\
\text { women }(71.5 \% \\
\text { aged } 25-34 \text { years) }\end{array}$ & $\begin{array}{l}\text { Prospective } \\
\text { cohort study } \\
\text { (AOB) } \\
2008\end{array}$ & $\begin{array}{l}<25 \text { th GW } \\
34 \text { th-36th } \\
\text { GW } \\
4 \text { months } \\
\text { postpartum }\end{array}$ & $\begin{array}{l}46 \% \text { of women } \\
\text { continued to drink } \\
\text { alcohol after } \\
\text { becoming aware } \\
\text { of their pregnancy; } \\
\text { usually low } \\
\text { ( }<1 \text { drink per occa- } \\
\text { sion on } \leq 7 \text { days per } \\
\text { week) to moderate } \\
\text { amounts of alcohol } \\
\text { ( } 1 \text { drink per occa- } \\
\text { sion on } \leq 7 \text { days } \\
\text { per week or } 2 \text { drinks } \\
\text { per occasion on } \\
\leq 3 \text { days per week); } \\
13 \% \text { continued } \\
\text { binge-drinking } \\
\text { behavior ( } 5 \text { or more } \\
\text { alcoholic drinks } \\
\text { per occasion) in } \\
\text { early pregnancy. }\end{array}$ & $\begin{array}{l}\text { Predictors for binge drinking prior } \\
\text { to recognition of pregnancy: } \\
\text { Low level of education ( } O R=3.61 \text { ); nulliparity } \\
(\mathrm{OR}=1.62) \text {; unplanned pregnancy } \\
(\mathrm{OR}=1.93) \text {; nicotine consumption ( } \mathrm{OR}=1.90 \text { ) } \\
\text { and binge drinking in the } 12 \text { months prior to } \\
\text { pregnancy }(\mathrm{OR}=10.83) \text {; low dispositional op- } \\
\text { timism ( } \mathrm{OR}=1.73) \text {. } \\
\text { Predictors for low to moderate amounts } \\
\text { of alcohol consumed after recognition } \\
\text { of pregnancy: } \\
\text { unplanned pregnancy }(\mathrm{OR}=1.91) \text {; pre-preg- } \\
\text { nancy } \mathrm{BMI}<25.0 \mathrm{~kg} / \mathrm{m}^{2}(\mathrm{OR}=1.41) \text {; nicotine } \\
\text { consumption }(\mathrm{OR}=1.90) \text { and binge drinking } \\
\text { in the } 12 \text { months prior to pregnancy } \\
(\mathrm{OR}=2.62 \text { ). }\end{array}$ \\
\hline
\end{tabular}


Table 2 Findings of empirical studies on factors influencing health-damaging behaviors and mental health problems by pregnant women. (Continued)

\begin{tabular}{|c|c|c|c|c|c|c|}
\hline Authors & Country & Sample & Study design & $\begin{array}{l}\text { Time of data } \\
\text { collection }\end{array}$ & Prevalence & Factors which influenced behaviors \\
\hline [61] & France & $\begin{array}{l}\mathrm{n}=18014 \text { pregnant } \\
\text { women }(14.2 \% \\
<25 \text { years, } 31.3 \% \\
\text { aged } 25-29 \text { years, } \\
33.3 \% \text { aged } \\
30-34 \text { years, } 21.3 \% \\
\text { aged } \geq 35 \text { years) }\end{array}$ & $\begin{array}{l}\text { Birth cohort } \\
\text { study } \\
\text { (ELFE study) }\end{array}$ & $\begin{array}{l}\text { No data } \\
\text { provided } \\
2011\end{array}$ & $\begin{array}{l}\text { Nicotine consump- } \\
\text { tion: } 21.7 \% \\
\text { alcohol consump- } \\
\text { tion: } 34.8 \% \text { (binge } \\
\text { drinking: } 3.4 \% \text { ) } \\
\text { Women with immi- } \\
\text { gration status were } \\
\text { less likely to con- } \\
\text { sume nicotine } \\
\text { ( } 8.8 \text { vs. } 21.9 \% \text { ) and } \\
\text { alcohol ( } 23.4 \text { vs. } \\
40.7 \% \text { ); there were } \\
\text { no differences } \\
\text { with regard to } \\
\text { binge drinking } \\
\text { ( } 2.9 \text { vs. } 3.3 \% \text { ). }\end{array}$ & $\begin{array}{l}\text { 1. Factors associated with continued nicotine } \\
\text { consumption: } \\
\text { general factors: age } \leq 25 \text { years; single } \\
\text { parenthood; irregular attendance at prenatal } \\
\text { care appointments. } \\
\text { Non-immigrant smokers had lower educa- } \\
\text { tional levels (completed secondary school: } \\
\text { OR=3.09) and were not employed } \\
\text { (OR=1.45); weaker or inverse correlation in } \\
\text { immigrant women (completed secondary } \\
\text { school: OR }=1.86 \text {; not employed: OR }=0.68 \text { ); } \\
\text { however psychological problems (OR=1.95) } \\
\text { and comorbid alcohol consumption } \\
\text { (OR=1.92) were more strongly correlated } \\
\text { with nicotine consumption in immigrant } \\
\text { women. } \\
2 . \text { Factors associated with continued alcohol } \\
\text { consumption: } \\
\text { general factors: women aged } \geq 25 \text { years; } \\
\text { higher levels of education and employment; } \\
\text { more than one previous birth. } \\
\text { Migration-specific factors: single parenthood } \\
\text { (OR = 2.12); psychological problems } \\
\text { (OR=1.46). } \\
\text { Binge-drinking was associated with age } \\
\geq 35 \text { years (OR }=1.89 \text { ) and higher educational } \\
\text { qualifications (OR = } 1.32 \text { ) in non-immigrant } \\
\text { women. } \\
\text { Migration-specific risk factors for binge- } \\
\text { drinking: single parenthood (OR }=2.78 \text { ); } \\
\text { nicotine consumption (OR }=4.04 \text { ). }\end{array}$ \\
\hline [62] & Ireland & $\begin{array}{l}\mathrm{n}=907 \text { pregnant } \\
\text { women }(25.9 \% \text { aged } \\
25-29 \text { years, } 36.8 \% \\
\text { aged } 30-34 \text { years, } \\
20.7 \% \text { aged } \\
35-39 \text { years })\end{array}$ & $\begin{array}{l}\text { Prospective } \\
\text { cohort study }\end{array}$ & $\begin{array}{l}\text { 10th to 14th } \\
\text { GW } \\
28 \text { th GW } \\
\text { Birth } \\
2010-2011\end{array}$ & $\begin{array}{l}30 \% \text { smoked in the } \\
\text { last six months prior } \\
\text { to the pregnancy; } \\
41 \% \text { of smokers } \\
\text { continued to smoke } \\
\text { during pregnancy. }\end{array}$ & $\begin{array}{l}\text { Pregnant smokers differed from non-smokers } \\
\text { with regard to the following factors: Irish } \\
\text { nationality }(O R=3.23) \text {; unplanned pregnancy } \\
(O R=1.90) \text {; younger age (for age } 30-39 \\
\text { years: } O R=0.50) \text {; alcohol consumption dur- } \\
\text { ing the first trimester of pregnancy } \\
(O R=3.38) \text { and previous history of consuming } \\
\text { illegal drugs }(O R=3.56) \text {. }\end{array}$ \\
\hline [63] & Ireland & $\begin{array}{l}\mathrm{n}=6725 \text { pregnant } \\
\text { women }\end{array}$ & $\begin{array}{l}\text { Cross- } \\
\text { sectional } \\
\text { study }\end{array}$ & $\begin{array}{l}\text { From the } \\
\text { 12th GW }\end{array}$ & $\begin{array}{l}5 \% \text { of pregnant } \\
\text { women drank } \\
\text { alcohol; of these, } \\
92 \% \text { drank less than } \\
5 \text { glasses of alcohol } \\
\text { per week; } 8 \% \text { drank } \\
\text { between } 6 \text { and } \\
\geq 20 \text { glasses of } \\
\text { alcohol per week. }\end{array}$ & $\begin{array}{l}\text { Pregnant women who continued to drink } \\
\text { alcohol in early pregnancy were characterized } \\
\text { by the following factors: } \\
\text { higher maternal age ( } 30 \text { to } 39 \text { years: } \\
O R=1.64 ;>40 \text { years: } O R=3.27 \text { ); Irish } \\
\text { nationality (OR=3.05); concurrent nicotine } \\
\text { consumption (OR=2.58), and previous his- } \\
\text { tory of consuming illegal drugs ( } O R=2.67 \text { ). }\end{array}$ \\
\hline [64] & $\begin{array}{l}\text { UK, } \\
\text { Ireland, } \\
\text { Austra- } \\
\text { lia, New } \\
\text { Zealand }\end{array}$ & $\mathrm{n}=17244$ women & $\begin{array}{l}\text { GUI, PRAMS } \\
\text { Ireland } \\
\text { and SCOPE }\end{array}$ & $\begin{array}{l}\text { GUI: } \\
\text { 2008-2009 } \\
\text { PRAMS: } \\
2012 \text { register } \\
\text { of births } \\
\text { SCOPE: } \\
\text { 15th and } \\
\text { 20th GW } \\
\text { 2004-2011 }\end{array}$ & $\begin{array}{l}\text { Alcohol consump- } \\
\text { tion: } 20 \% \text { (GUI) and } \\
\text { up to } 82 \% \text { (SCOPE); } \\
30 \% \text { (SCOPE) and } \\
\text { up to } 85 \% \text { (PRAMS) } \\
\text { of women reported } \\
\text { consuming one } \\
\text { to two glasses of } \\
\text { alcohol per week. }\end{array}$ & $\begin{array}{l}\text { Predictor for alcohol consumption: } \\
\text { concurrent nicotine consumption during } \\
\text { pregnancy: } \\
\text { SCOPE: } R R=1.17 \text {; GUI: RR=1.50; } \\
\text { PRAMS: RR = } 1.42 \text {. }\end{array}$ \\
\hline
\end{tabular}


Table 2 Findings of empirical studies on factors influencing health-damaging behaviors and mental health problems by pregnant women. (Continued)

\begin{tabular}{|c|c|c|c|c|c|c|}
\hline Authors & Country & Sample & Study design & $\begin{array}{l}\text { Time of data } \\
\text { collection }\end{array}$ & Prevalence & Factors which influenced behaviors \\
\hline [65] & Australia & $\begin{array}{l}n=1591 \text { pregnant } \\
\text { women (61.3\% aged } \\
25-29 \text { years) }\end{array}$ & $\begin{array}{l}\text { Prospective } \\
\text { cohort study } \\
\text { (ALSWH) }\end{array}$ & $\begin{array}{l}\text { No precise } \\
\text { data given } \\
2000,2003 \text {, } \\
2006\end{array}$ & $\begin{array}{l}72.7 \% \text { of women } \\
\text { drank alcohol } \\
\text { during pregnancy } \\
\text { (at least } 1-2 \text { drinks } \\
\text { per occasion); } 1.8 \% \\
\text { of women smoked } \\
\text { during pregnancy. } \\
8.2 \% \text { of women } \\
\text { concurrently con- } \\
\text { sumed nicotine } \\
\text { and alcohol. }\end{array}$ & $\begin{array}{l}\text { Women who drank more than } 2 \text { alcoholic } \\
\text { drinks per day or drank more than once a } \\
\text { week were more likely to reduce their alcohol } \\
\text { consumption. } \\
\text { Quitting smoking was more difficult if the } \\
\text { women had financial problems ( } R R=0.78 \text { ); } \\
\text { experienced violence by their partner } \\
\text { (RR }=0.76 \text { ); had other children ( } R R=0.87 \text { ), } \\
\text { and if their pre-pregnancy nicotine consump- } \\
\text { tion was more than } 10 \text { cigarettes per day } \\
\text { (RR }=0.75 \text { ). } \\
\text { The decrease in the concurrent consumption } \\
\text { of nicotine and alcohol was sign. higher for } \\
\text { women who had attended school for at least } \\
12 \text { years (RR }=1.5-1.6) \text {; who drank alcohol at } \\
\text { least } 1-2 \text { days/week prior to being pregnant } \\
\text { (RR }=1.5-1.6) \text {, and who consumed } 3 \text { or more } \\
\text { alcoholic drinks per occasion (RR }=1.6-1.8 \text { ) } \\
\text { and sign. lower for women who smoked more } \\
\text { than } 10 \text { cigarettes; for women with other } \\
\text { children (RR }=0.79 \text { ), and for women with } \\
\text { financial problems (RR }=0.74 \text { ), and women } \\
\text { who had experienced partner violence } \\
\text { (RR }=0.71 \text { ). }\end{array}$ \\
\hline [66] & Sweden & $\begin{array}{l}\mathrm{n}=1594 \text { pregnant } \\
\text { women }(32.8 \% \text { aged } \\
25-29 \text { years, } 34.2 \% \\
\text { aged } 30-34 \text { years) }\end{array}$ & $\begin{array}{l}\text { Cross- } \\
\text { sectional } \\
\text { study }\end{array}$ & $\begin{array}{l}\geq 18 \text { th GW } \\
2009-2010\end{array}$ & $\begin{array}{l}6 \% \text { of pregnant } \\
\text { women drank at } \\
\text { least once a week. }\end{array}$ & $\begin{array}{l}\text { Factors associated with continued alcohol } \\
\text { consumption: } \\
\text { increased maternal age }(30-34 \text { years: } \\
O R=4.54 ; 35-39 \text { years: } O R=8.51 ; \geq 40 \text { years: } \\
O R=11.32) \text {; place of residence (large city: } \\
O R=1.69) \text {; daily consumption of nicotine pri- } \\
\text { or to pregnancy }(O R=3.76) \text {; less social sup- } \\
\text { port }(O R=0.86) \text {; drinking habits }(O R=0.86) \text {; } \\
\text { social reasons for drinking }(O R=1.12) \text {. }\end{array}$ \\
\hline [67] & $\begin{array}{l}15 \text { coun- } \\
\text { tries }\end{array}$ & $\begin{array}{l}\mathrm{n}=8344 \text { pregnant } \\
\text { women and mothers } \\
\text { of infants under } \\
\text { the age of one year }\end{array}$ & $\begin{array}{l}\text { Cross- } \\
\text { sectional } \\
\text { study }\end{array}$ & $\begin{array}{l}\text { No data } \\
\text { 2011-2012 }\end{array}$ & $\begin{array}{l}35.3 \% \text { of women } \\
\text { smoked prior to } \\
\text { the pregnancy and } \\
26.2 \% \text { smoked } \\
\text { during pregnancy. }\end{array}$ & $\begin{array}{l}\text { Factors associated with continued consump- } \\
\text { tion of nicotine: } \\
\text { no spouse or partner }(\mathrm{aOR}=1.75) \text {; low level } \\
\text { of education ( } \mathrm{aOR}=3.64) \text {; being a housewife } \\
\text { (aOR=1.43); other children }(\mathrm{aOR}=1.24) \text {; } \\
\text { unplanned pregnancy }(\mathrm{aOR}=1.31) \text {; did not } \\
\text { take folic acid supplements }(\mathrm{aOR}=1.59 \text { ); } \\
\text { low health literacy ( } \mathrm{aOR}=1.43 \text { ) }\end{array}$ \\
\hline [68] & $\begin{array}{l}15 \text { coun- } \\
\text { tries }\end{array}$ & $\begin{array}{l}n=4295 \text { pregnant } \\
\text { women }\end{array}$ & $\begin{array}{l}\text { Cross- } \\
\text { sectional } \\
\text { study }\end{array}$ & $\begin{array}{l}\text { No data } \\
2011-2012\end{array}$ & $\begin{array}{l}34.5 \% \text { of women } \\
\text { smoked prior to } \\
\text { the pregnancy and } \\
26.4 \% \text { continued } \\
\text { to smoke during } \\
\text { pregnancy. }\end{array}$ & $\begin{array}{l}\text { Factors associated with continued consump- } \\
\text { tion of nicotine: } \\
\text { Women who continued to smoke during } \\
\text { pregnancy were more likely to be depressed } \\
\text { than women who quit smoking } \\
\text { ( } 32.5 \text { vs. } 18.9 \% ; p<0.001) \text {. } \\
\text { Predictors for continued consumption } \\
\text { of nicotine: } \\
\text { lower level of education (OR }=4.46) \text {; } \\
\text { depression }(O R=2.02) \text {. }\end{array}$ \\
\hline \multicolumn{7}{|c|}{ Diet and weight gain/obesity } \\
\hline [69] & $\begin{array}{l}\text { Nether- } \\
\text { lands }\end{array}$ & $\begin{array}{l}n=144 \text { pregnant } \\
\text { women } \\
(M=31.2 \text { years; } \\
S D=4.7)\end{array}$ & $\begin{array}{l}\text { Prospective } \\
\text { study }\end{array}$ & $\begin{array}{l}\text { 30th GW } \\
(M=30.4 \mathrm{GW} ; \\
S D=1.9) \\
2003-2007\end{array}$ & $\begin{array}{l}38 \% \text { of pregnant } \\
\text { women gained } \\
\text { more weight than } \\
\text { recommended } \\
\text { (particularly } \\
\text { overweight and } \\
\text { obese women). }\end{array}$ & $\begin{array}{l}\text { Predictors of excessive weight gain: } \\
\text { Overweight pre-pregnancy }(\mathrm{OR}=6.33) \text {; } \\
\text { low level of physical activity }(\mathrm{OR}=3.96) ; \\
\text { elevated food intake ( } \mathrm{OR}=3.14) \text {. } \\
\text { The risk was reduced when women had } \\
\text { more than nine hours' sleep daily }(\mathrm{OR}=0.35 \text { ). }\end{array}$ \\
\hline
\end{tabular}


Table 2 Findings of empirical studies on factors influencing health-damaging behaviors and mental health problems by pregnant women. (Continued)

\begin{tabular}{|c|c|c|c|c|c|c|}
\hline Authors & Country & Sample & Study design & $\begin{array}{l}\text { Time of data } \\
\text { collection }\end{array}$ & Prevalence & Factors which influenced behaviors \\
\hline [70] & USA & $\begin{array}{l}\mathrm{n}=1100 \text { pregnant } \\
\text { women }\end{array}$ & $\begin{array}{l}\text { Cross- } \\
\text { sectional } \\
\text { study (WISH) }\end{array}$ & $\begin{array}{l}\text { 32nd to 36th } \\
\text { GW }\end{array}$ & $\begin{array}{l}14 \% \text { of pregnant } \\
\text { women had insuffi- } \\
\text { cient weight gain } \\
\text { and } 53 \% \text { had exces- } \\
\text { sive weight gain. }\end{array}$ & $\begin{array}{l}\text { Predictors of excessive weight gain: } \\
\text { overweight pre-pregnancy }(O R=2.26) \text {; } \\
\text { nullipara vs. multipara }(O R=1.4) \text {. } \\
\text { Predictors of insufficient weight gain: } \\
\text { chronic or gestational diabetes }(O R=2.70) \text {; } \\
\text { higher or lower consumption of dairy } \\
\text { products ( } O R=1.74) \text {; nullipara vs. multipara } \\
(O R=0.62) \text {. }\end{array}$ \\
\hline [71] & USA & $\begin{array}{l}\mathrm{n}=770 \text { Hispanic } \\
\text { pregnant women } \\
\text { (35\%<20 years; } \\
37.4 \% \text { aged } \\
20-24 \text { years; } 17.9 \% \\
\text { aged } 25-29 \text { years) }\end{array}$ & $\begin{array}{l}\text { Prospective } \\
\text { cohort study }\end{array}$ & $M=15$ th GW & $\begin{array}{l}22 \% \text { of pregnant } \\
\text { women had insuffi- } \\
\text { cient weight gain } \\
\text { and } 45 \% \text { had exces- } \\
\text { sive weight gain. }\end{array}$ & $\begin{array}{l}\text { Predictors for excessive weight gain: } \\
\text { women aged more than } 30 \text { years }(\mathrm{OR}=2.5) \\
\text { compared to women aged } 20-24 \text { years; } \\
\text { overweight compared to normal weight } \\
(\mathrm{OR}=2.2) \text {. } \\
\text { Lower risk for women who had already born } \\
\text { more than two children }(\mathrm{OR}=0.2) \text { and who } \\
\text { had resided less than } 10 \text { years in the USA } \\
(\mathrm{OR}=0.5) \text {. }\end{array}$ \\
\hline [72] & $\begin{array}{l}\text { Nether- } \\
\text { lands }\end{array}$ & $\begin{array}{l}\mathrm{n}=6959 \text { pregnant } \\
\text { women } \\
\text { ( } \mathrm{M}=30.3 \text { years; } \\
\text { range: } \\
20.4-37.9 \text { years })\end{array}$ & $\begin{array}{l}\text { Prospective } \\
\text { cohort study } \\
\text { (Generation R } \\
\text { study) }\end{array}$ & $\begin{array}{l}\text { No informa- } \\
\text { tion provided } \\
\text { on the time- } \\
\text { point in the } \\
\text { when data was } \\
\text { collected } \\
2001-2005\end{array}$ & $\begin{array}{l}16.2 \% \text { of pregnant } \\
\text { women were } \\
\text { underweight, } \\
55.8 \% \text { of pregnant } \\
\text { women were nor- } \\
\text { mal weight, } 19.2 \% \\
\text { were overweight, } \\
8.8 \% \text { were obese } \\
44.5 \% \text { of pregnant } \\
\text { women had exces- } \\
\text { sive weight gain. }\end{array}$ & $\begin{array}{l}\text { Factors associated with obesity: } \\
\text { low level of maternal education ( } O R=2.48) \text {; } \\
\text { household income }<1600 \text { Euros }(O R=1.36) \text {; } \\
\text { multipara ( } O R=1.68 \text { ). } \\
\text { Risk factors for excessive weight gain: } \\
\text { European ethnicity (Ref.: non-European } \\
\text { ethnicity, } O R=0.78) \text {; nulliparity (Ref.: multi- } \\
\text { para, } O R=0.71) \text {; higher energy intake } \\
(O R=1.13) \text {; nicotine consumption during } \\
\text { pregnancy }(O R=2.08) \text {; BMl of the father was } \\
\text { a risk factor for maternal obesity }(O R=1.53) \\
\text { and for excessive weight gain }(O R=1.12) \text {. }\end{array}$ \\
\hline [73] & USA & $\begin{array}{l}\mathrm{n}=94 \text { pregnant } \\
\text { women } \\
\text { ( } 73 \% \text { aged } \\
<25 \text { years) }\end{array}$ & $\begin{array}{l}\text { Prospective } \\
\text { cohort study }\end{array}$ & $\begin{array}{l}M=21 \text { st GW } \\
2008\end{array}$ & $\begin{array}{l}60 \% \text { of pregnant } \\
\text { women were over- } \\
\text { weight and } 41 \% \\
\text { of pregnant women } \\
\text { had excessive } \\
\text { weight gain. }\end{array}$ & $\begin{array}{l}\text { Predictors for excessive weight gain: } \\
\text { overweight or obese pre-pregnancy } \\
(\mathrm{BMI} \geq 25 \text { : } \mathrm{aOR}=4.20) \text {; nulliparity } \\
(\mathrm{aOR}=3.35) \text {. } \\
\text { Less than } 2 \mathrm{~h} \text { daily television viewing } \\
(\mathrm{aOR}=0.18) \text { and regular physical activity } \\
(\mathrm{aOR}=0.35) \text { were associated with a lower risk } \\
\text { of excessive weight gain. }\end{array}$ \\
\hline [74] & USA & $\begin{array}{l}n=3006 \text { pregnant } \\
\text { women } \\
(M=27.0 \text { years; } \\
S D=5.9)\end{array}$ & $\begin{array}{l}\text { Prospective } \\
\text { cohort study }\end{array}$ & $\begin{array}{l}\text { 34th GW } \\
\text { 2009-2011 }\end{array}$ & $\begin{array}{l}78.7 \% \text { of over- } \\
\text { weight, } 65 \% \\
\text { of obese and } 42.4 \% \\
\text { of normal weight } \\
\text { pregnant women } \\
\text { exceeded recom- } \\
\text { mendations on } \\
\text { weight gain. }\end{array}$ & $\begin{array}{l}\text { Predictors for excessive weight gain: } \\
\text { older age ( } 30-36 \text { years: } \mathrm{aOR}=1.45) \text {; } \\
\text { unmarried and living with partner } \\
(\mathrm{aOR}=1.48 \text { ) or with no partner ( } \mathrm{aOR}=1.46 \text { ); } \\
\text { overweight }(\mathrm{aOR}=5.11 \text { ) or obese } \\
(\mathrm{aOR}=2.35) \text {; smoking status }(\mathrm{aOR}=1.49) \text {. } \\
\text { Physical activity of at least } 150 \text { min per week } \\
\text { reduced risk of excessive weight gain. }\end{array}$ \\
\hline [75] & USA & $\begin{array}{l}\mathrm{n}=2994 \\
(\mathrm{n}=855 \text { nullipara; } \\
\mathrm{n}=2139 \text { multipara) }\end{array}$ & $\begin{array}{l}\text { Cross- } \\
\text { sectional } \\
\text { study } \\
\text { (IFPS II) }\end{array}$ & $\begin{array}{l}\text { Prior to } \\
\text { the birth of } \\
\text { the child } \\
2005-2007\end{array}$ & & $\begin{array}{l}\text { Predictors for excessive weight gain/obesity: } \\
\text { Nullipara gained significantly more weight } \\
\text { than multipara; multipara were far more likely } \\
\text { to be overweight or obese than nullipara. }\end{array}$ \\
\hline [76] & USA & $\begin{array}{l}n=279 \text { pregnant } \\
\text { women } \\
(M=27.0 \text { years; } \\
S D=5.9)\end{array}$ & $\begin{array}{l}\text { Prospective } \\
\text { longitudinal } \\
\text { studies }\end{array}$ & $\begin{array}{l}\mathrm{t}_{1}: 10 \text { th to } \\
22 \mathrm{nd} \mathrm{GW} \\
(M=16.6 \mathrm{GW} ; \\
S D=4.4) \\
\mathrm{t}_{2}: 21 \mathrm{st} \text { to } \\
30 \text { th GW } \\
(M=26.1 \mathrm{GW} ; \\
S D=3.8) \\
\mathrm{t}_{3}: \text { after } 30 \text { th } \\
\mathrm{GW}(M=34.1 \\
\mathrm{GW} ; S D=2.4)\end{array}$ & & $\begin{array}{l}\text { Pregnancy-specific stress was a predictor } \\
\text { for the following health-damaging behaviors: } \\
\text { nicotine consumption }(B=0.22) \text {; caffeine } \\
\text { consumption }(B=0.16) \text {; unhealthy diet } \\
(B=0.29) \text {. }\end{array}$ \\
\hline
\end{tabular}


Table 2 Findings of empirical studies on factors influencing health-damaging behaviors and mental health problems by pregnant women. (Continued)

\begin{tabular}{|c|c|c|c|c|c|c|}
\hline Authors & Country & Sample & Study design & $\begin{array}{l}\text { Time of data } \\
\text { collection }\end{array}$ & Prevalence & Factors which influenced behaviors \\
\hline [77] & $\begin{array}{l}\text { UK, } \\
\text { Canada }\end{array}$ & $\begin{array}{l}n=2282 \text { pregnant } \\
\text { women } \\
(M=29.6 \text { years; } \\
S D=5.0)\end{array}$ & $\begin{array}{l}\text { Cross- } \\
\text { sectional } \\
\text { study }\end{array}$ & $\begin{array}{l}\text { No data } \\
\text { provided }\end{array}$ & & $\begin{array}{l}\text { Predictors of an unhealthy diet: } \\
\text { Immigrant status, unmarried women } \\
\text { in a permanent relationship; nulliparity; } \\
\text { low level of physical activity; smoker; } \\
\text { higher anxiety scores; lack of familial support. }\end{array}$ \\
\hline [78] & Italy & $\begin{array}{l}\mathrm{n}=2189 \text { pregnant } \\
\text { women } \\
(\mathrm{M}=33 \text { years) }\end{array}$ & $\begin{array}{l}\text { Cross- } \\
\text { sectional } \\
\text { study }\end{array}$ & $\begin{array}{l}\text { No data on } \\
\text { the time-point } \\
\text { of data collec- } \\
\text { tion during } \\
\text { pregnancy } \\
2012\end{array}$ & $\begin{array}{l}23.5 \% \text { of women } \\
\text { took folic acid } \\
\text { supplements } \\
\text { pre-pregnancy }\end{array}$ & 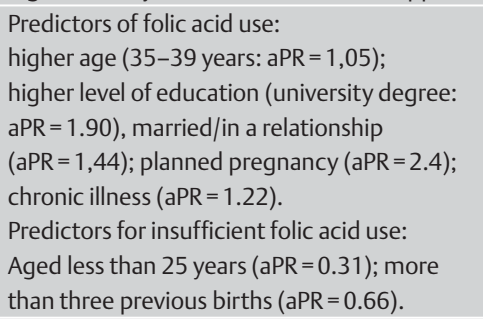 \\
\hline [79] & Norway & $\begin{array}{l}\mathrm{n}=225000 \text { pregnant } \\
\text { women (age at } \\
\text { delivery: } 72.4 \% \\
\text { aged } 25-34 \text { years) }\end{array}$ & $\begin{array}{l}\text { Prospective } \\
\text { cohort study } \\
\text { (MoBa) }\end{array}$ & $\begin{array}{l}17 \text { th to } 18 \text { th } \\
\text { GW } \\
2000-2003\end{array}$ & $\begin{array}{l}11.8 \% \text { women took } \\
\text { folic acid supple- } \\
\text { ments two months } \\
\text { prior to pregnancy, } \\
\text { increasing to } 46.9 \% \\
\text { in the third trimes- } \\
\text { ter of pregnancy }\end{array}$ & $\begin{array}{l}\text { Predictors of folic acid use: } \\
\text { higher level of education (aRR=4.1); } \\
\text { higher age (age from } 25-34 \text { years: } a R R=1.6 \text { ); } \\
\text { higher income (aRR=1.3); married } \\
\text { (aRR=2.4); planned pregnancy. } \\
\text { Predictors for insufficient intake of folic acid } \\
\text { supplements: } \\
\text { Nicotine consumption ( } a R R=0.6 \text { ); } \\
\text { number of previous births }(<2: a R R=0.8 \text { ). }\end{array}$ \\
\hline [80] & $\begin{array}{l}\text { Den- } \\
\text { mark }\end{array}$ & $\begin{array}{l}\mathrm{n}=60892 \text { pregnant } \\
\text { women }\end{array}$ & $\begin{array}{l}\text { Prospective } \\
\text { cohort study } \\
\text { (DNBC) }\end{array}$ & $\begin{array}{l}\text { 16th and } 18 \text { th } \\
\text { GW } \\
6 \text { and } 18 \\
\text { months } \\
\text { postpartum } \\
1996-2002\end{array}$ & $\begin{array}{l}4.3 \% \text { underweight; } \\
68.3 \% \text { normal } \\
\text { weight; } 19.5 \% \\
\text { overweight; } 7.9 \% \\
\text { obese } \\
21.9 \% \text { of the total } \\
\text { sample had exces- } \\
\text { sive weight gain. } \\
\text { Insufficient weight } \\
\text { gain was more likely } \\
\text { among overweight } \\
(17.7 \% \text { and obese } \\
\text { women (42.0\%). }\end{array}$ & $\begin{array}{l}\text { Underweight, overweight and obese preg- } \\
\text { nant women were slightly younger and more } \\
\text { likely to be multipara compared to pregnant } \\
\text { women who were normal weight. } \\
\text { Determinants of insufficient weight gain } \\
\text { (<10 kilos): } \\
\text { higher age ( } \geq 35 \text { years); lower social status; } \\
\text { short stature; multipara; smoking more than } \\
10 \text { cigarettes per day, and drinking more than } \\
\text { three alcoholic drinks during pregnancy. } \\
\text { Determinants of higher to excessive weight } \\
\text { gain ( } 16-19 \text { or more than } 20 \text { kilos): } \\
\text { Aged less than } 30 \text { years and primiparity; } \\
\text { low social status; taller; smoking less than } \\
10 \text { cigarettes per day; no alcohol consump- } \\
\text { tion during pregnancy; lack of exercise. }\end{array}$ \\
\hline [81] & UK & $\begin{array}{l}\mathrm{n}=12053 \text { pregnant } \\
\text { women }(39.1 \% \text { aged } \\
<20 \text { years, } 39 \% \text { aged } \\
20-24 \text { years, } 17.9 \% \\
\text { aged } 25-29 \text { years })\end{array}$ & $\begin{array}{l}\text { Prospective } \\
\text { cohort study } \\
\text { (ALSPAC) }\end{array}$ & $\begin{array}{l}\text { 8th, 18th and } \\
\text { 32nd GW } \\
1991-1992\end{array}$ & & $\begin{array}{l}\text { Predictors of diet: } \\
\text { Health-conscious diet correlated positively } \\
\text { with higher education and age. There was } \\
\text { a negative correlation with increased parity, } \\
\text { single parenthood; unemployment; smoking } \\
\text { status and being overweight pre-pregnancy. } \\
\text { Traditional diet (e.g. consumption of red } \\
\text { meat, potatoes, green vegetables, etc.) was } \\
\text { associated with physical activity; being over- } \\
\text { weight pre-pregnancy, and increased parity. } \\
\text { The consumption of high-fat processed foods } \\
\text { (e.g. pizza, chips) was associated with youn- } \\
\text { ger age; low level of education; financial } \\
\text { difficulties; smoking status; increased parity. } \\
\text { Consumption of high-sugar foods was } \\
\text { associated with younger age; ethnicity, } \\
\text { higher anxiety scores; multiparity. }\end{array}$ \\
\hline [82] & France & $\begin{array}{l}n=903 \text { pregnant } \\
\text { women } \\
(M=31.7 \text { years; } \\
S D=4.7)\end{array}$ & $\begin{array}{l}\text { Prospective } \\
\text { cohort study } \\
\text { (NutriNet- } \\
\text { Santé study) }\end{array}$ & $\begin{array}{l}31 \% \text { in the first } \\
\text { trimester } \\
\text { of pregnancy, } \\
36 \% \text { in the } \\
\text { second tri- } \\
\text { mester and } \\
33 \% \text { in the } \\
\text { third trimester } \\
2009\end{array}$ & $\begin{array}{l}64.9 \% \text { of pregnant } \\
\text { women took } \\
\text { dietary supple- } \\
\text { ments }(45 \% \text { folic } \\
\text { acid, } 42.1 \% \\
\text { iron and } 15.5 \% \\
\text { vitamin D supple- } \\
\text { ments). }\end{array}$ & $\begin{array}{l}\text { Factors associated with use of dietary } \\
\text { supplements: } \\
\text { higher age }(\mathrm{aOR}=1.06 \text { ) and income } \\
\text { (>3130 Euros per month: } \mathrm{aOR}=2.09 \text { ). } \\
\text { Factors associated with no use of dietary } \\
\text { supplements: } \\
\text { occupational category (working class } \\
\text { aOR }=0.42 \text {; unemployed: } \mathrm{aOR}=0.45 \text { ); } \\
\text { multiparity (aOR=0.52). }\end{array}$ \\
\hline
\end{tabular}


Table 2 Findings of empirical studies on factors influencing health-damaging behaviors and mental health problems by pregnant women. (Continued)

\begin{tabular}{|c|c|c|c|c|c|c|}
\hline Authors & Country & Sample & Study design & $\begin{array}{l}\text { Time of data } \\
\text { collection }\end{array}$ & Prevalence & Factors which influenced behaviors \\
\hline [83] & $\begin{array}{l}\text { Austra- } \\
\text { lia, New } \\
\text { Zealand } \\
\text { and } \\
\text { Ireland }\end{array}$ & $\begin{array}{l}n=1950 \text { nullipara } \\
\text { (Australia } n=475 \\
M=24.3 \text { years; } \\
S D=5.0 \text { ); (New } \\
\text { Zealand } n=264 \\
M=30.4 \text { years; } \\
S D=4.9 \text { ); (Ireland } \\
n=1211 ; \\
M=29.9 \text { years; } \\
S D=4.5 \text { ) }\end{array}$ & SCOPE & $\begin{array}{l}\text { 14th to } 16 \text { th } \\
\text { GW }\end{array}$ & $\begin{array}{l}17 \% \text { of pregnant } \\
\text { women had } \\
\text { sufficient weight } \\
\text { gain, } 74 \% \text { of preg- } \\
\text { nant women had } \\
\text { excessive weight } \\
\text { gain and } 9 \% \text { had } \\
\text { insufficient weight } \\
\text { gain. }\end{array}$ & 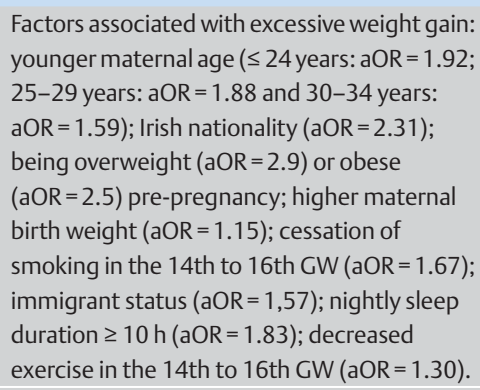 \\
\hline [84] & USA & $\begin{array}{l}n=1777 \text { pregnant } \\
\text { women } \\
(M=32.4 \text { years; } \\
S D=4.9) \text {; } \\
49 \% \text { nullipara }\end{array}$ & $\begin{array}{l}\text { Prospective } \\
\text { study }\end{array}$ & $\begin{array}{l}\text { 1st prenatal } \\
\text { appointment } \\
(\mathrm{M}=11.7 \mathrm{GW} \text {; } \\
\mathrm{SD}=3.1) \\
\text { 26th-28th } \\
\mathrm{GW}\end{array}$ & & $\begin{array}{l}\text { Predictors for poorer quality diet: } \\
\text { younger maternal age; lower level } \\
\text { of education; higher BMI pre-pregnancy; } \\
\text { higher number of previous births. }\end{array}$ \\
\hline [85] & USA & $\begin{array}{l}n=795 \text { pregnant } \\
\text { women }(n=355 \text { aged } \\
\leq 25 \text { years; } \\
n=20-35 \text { years; } \\
n=63 \text { aged } \\
\geq 35 \text { years })\end{array}$ & $\begin{array}{l}\text { Cross- } \\
\text { sectional } \\
\text { study }\end{array}$ & 2003-2012 & & $\begin{array}{l}\text { Predictors of an unhealthy diet: } \\
\text { being overweight (aOR=3.8) or obese } \\
\text { (aOR=5.4) before pregnancy. } \\
\text { Significantly higher HEl-2010 scores were } \\
\text { reported for women with higher incomes; } \\
\text { higher level of education; aged } \leq 35 \text { years; } \\
\text { married women, and non-smokers. }\end{array}$ \\
\hline [86] & $\begin{array}{l}\text { Nether- } \\
\text { lands }\end{array}$ & $\begin{array}{l}\mathrm{n}=6959 \text { pregnant } \\
\text { women }(4.0 \% \text { aged } \\
<20 \text { years, } 43.1 \% \\
\text { aged } 20-29.9 \text { years, } \\
37.9 \% \text { aged } \\
30-35 \text { years; } 15.0 \% \\
\text { aged }>30 \text { years })\end{array}$ & $\begin{array}{l}\text { Prospective } \\
\text { cohort study } \\
\text { (Generation R } \\
\text { study) }\end{array}$ & $\begin{array}{l}\text { Median: } \\
\text { 14.4 GW } \\
2002-2006\end{array}$ & $\begin{array}{l}37 \% \text { of pregnant } \\
\text { women took } \\
\text { sufficient folic acid } \\
\text { supplements } \\
\text { pre-pregnancy. }\end{array}$ & $\begin{array}{l}\text { Predictors for insufficient intake of folic acid } \\
\text { supplements: } \\
\text { low level of education }(\mathrm{aOR}=2.5) \text {; younger } \\
\text { age }(<20 \text { years: } \mathrm{aOR}=1.5 ; 20-29.9 \text { years } \\
\mathrm{aOR}=1.4) \text {; single }(\mathrm{aOR}=2.0) \text {; } \text { non-Western } \\
\text { European ethnicity }(\mathrm{aOR}=3.5) \text {; } \text { nicotine } \\
(\mathrm{aOR}=2.3 \text { ) and alcohol consumption during } \\
\text { pregnancy }(\mathrm{aOR}=1.5) \text {; unplanned pregnancy } \\
(\mathrm{aOR}=9.5) \text {; multiparity }(\mathrm{aOR}=1.6) \text {. }\end{array}$ \\
\hline [87] & $\begin{array}{l}\text { New } \\
\text { Zealand }\end{array}$ & $\begin{array}{l}\mathrm{n}=5664 \text { pregnant } \\
\text { women (median: } \\
31 \text { years; range: } \\
15 \text { to } 47 \text { years) }\end{array}$ & $\begin{array}{l}\text { Prospective } \\
\text { cohort study } \\
\text { (Growing up } \\
\text { in New } \\
\text { Zealand) }\end{array}$ & $\begin{array}{l}\text { Third } \\
\text { trimester of } \\
\text { pregnancy } \\
\text { 2009-2010 }\end{array}$ & & $\begin{array}{l}\text { Predictors of diet: } \\
\text { Junk food diet was associated with younger } \\
\text { age; lower level of education; poorer } \\
\text { self-rated health, and depressive symptoms; } \\
\text { continued consumption of nicotine and alco- } \\
\text { hol and no intake of folic acid supplements. } \\
\text { Higher scores for a healthy diet were asso- } \\
\text { ciated with increasing age (> } 40 \text { years); better } \\
\text { self-rated health; lower pre-pregnancy BMl; } \\
\text { physical activity, and not smoking. } \\
\text { Traditional diet (consumption of milk, white } \\
\text { bread, etc.) was associated with younger age; } \\
\text { lower levels of education; socio-economic } \\
\text { disadvantage; continued consumption } \\
\text { of nicotine; no folic acid supplement use; } \\
\text { unplanned pregnancy; multiparity. } \\
\text { Higher levels of consumption of high-protein } \\
\text { foods was associated with higher age; better } \\
\text { self-rated health; abstaining from smoking; } \\
\text { physical activity; nulliparity. }\end{array}$ \\
\hline
\end{tabular}

ALSPAC: Avon Longitudinal Study of Parents and Children. ALSWH: Australian Longitudinal Study on Woman's Health. AOB: All Our Babies. aOR: adjusted odds ratio. aPR: adjusted prevalence ratio. B: coefficient. DNBC: Danish National Birth Cohort. GUI: Growing up in Ireland. GW: week of gestation. HEl-2010: Healthy Eating Index 2010. IFPS II: Infant Feeding Practices Study II. M: mean. MoBa: Norwegian Mother and Child Cohort Study. NHANES: National Health and Nutrition Examination Survey. PRAMS: Pregnancy Risk Assessment Monitoring System. OR: odds ratio. PAD study: Pregnancy, Anxiety and Depression Study. RR: relative risk. SCOPE: Screening for Pregnancy Endpoints. SD: standard deviation. sign.: significant. WISH: Woman and Infants Starting Healthy. 
ing during pregnancy [65]. Nicotine consumption was often also associated with concurrent consumption of alcohol and illegal drugs, either prior to or during pregnancy [51,58,62,65]. Pregnant women who smoked were also more likely to have an unplanned pregnancy $[51,62,67]$ and these women also attended routine antenatal medical checkups less often or only in the advanced stage of pregnancy compared to non-smokers [32,35,61]. Multipara were found to be a group at particular risk of continuing to smoke during pregnancy compared to women who were pregnant with their first child or women who had previously given birth to only one child [32,35,56-57,65,67].

In contrast to maternal nicotine consumption the consumption of low to moderate amounts of alcohol during pregnancy is a risk factor which was more likely to affect women with a higher socio-economic status and women who were older (aged $>30$ years) $[34,61,63,66]$. Skagerstróm et al. [36], who summarized the findings of 14 studies published between 2002 and 2009, did not come to a definitive conclusion with respect to the impact of the pregnant woman's social status on her prenatal consumption of alcohol. Melchior et al. [61] showed a number of different predictors for occasional excessive alcohol consumption during pregnancy depending on the pregnant woman's immigrant status. Binge-drinking behavior during pregnancy was only associated with non-immigrant pregnant women who were older and had higher educational qualifications.

Prior experience of abuse or violence by the intimate partner or other persons is considered to be an important psychological factor associated with the continued consumption of alcohol during pregnancy [36]. However, Skagerstróm et al. [36] only identified three studies which looked at this issue (cf. $\bigcirc$ Table 1). Powers et al. [65] reported that the expectant mother's experience of violence by her partner was more closely associated with continued alcohol and nicotine consumption. Beijers et al. [52] found that critical life events such as crime-related events (e.g. involved in an accident), conflicts with her partner or pregnancy-specific events (e.g. bleeding, prenatal diagnosis to investigate potential abnormalities/malformations) could also substantially increase the risk of continued alcohol consumption during pregnancy (cf. - Table 2), although the authors found no correlation between prenatal alcohol consumption and increased depression or anxiety scores. It is possible that consuming alcohol or nicotine in this context could be viewed as a simple, albeit maladaptive, strategy used by affected women to deal with negative emotions and cope with traumatic events [36,52], particularly if the affected women do not receive support from their partner to process emotional stresses [65].

To what extent pregnant women managed to stop drinking alcohol depended, essentially, on the amount of alcohol and the frequency with which it was consumed before she became pregnant [36]. Women who drank more than two alcoholic drinks per week or more than five alcoholic drinks per occasion (binge drinking) before they became pregnant, found it significantly more difficult to abstain entirely from drinking alcohol during pregnancy $[55,60,65]$. The same applied to pregnant women who had additionally smoked before or during pregnancy [55, 63-66]. Two of the studies found a correlation between continued maternal alcohol consumption and unplanned pregnancy $[55,60]$; it was not clear, however, whether nullipara or multipara found it more difficult to abstain from drinking alcohol [36]. McDonald et al. [60] reported, for example, that women who had not previously given birth were more likely to binge drink during the early stages of pregnancy, i.e., before they knew they were pregnant. In contrast, continued low to moderate alcohol consumption during pregnancy was more common among women who had already previously given birth to several children $[36,61,65]$.

\section{Dietary Habits and Weight Gain}

$\nabla$

Dietary habits and sufficient weight gain during pregnancy play an important role in the physical and mental health of expectant women as well as for the development and the later health of the child [90-91]. The rapid growth and neural development of the unborn child places special demands on the nutrition ingested by pregnant women $[4,92]$. While the energy needs of pregnant women do not change much over the course of pregnancy, the pregnant woman has an increased need of micronutrients (e.g. vitamins and minerals) [91]. When pregnant women are malnourished or have nutritional deficiencies this can be due to an undersupply or oversupply of calories along with a corresponding weight gain or it may be due to the inadequate quality of the food consumed and a low supply of micronutrients [4]. In addition to dietary habits, the pregnant woman's pre-pregnancy weight can also affect weight gain during pregnancy and have a lasting impact on the further course of her pregnancy and on the course of the birth. It is therefore recommended that women of normal weight (BMI 18.5-24.9 $\mathrm{kg} / \mathrm{m}^{2}$ ) should not gain more than a maximum of 16 kilos over the course of the pregnancy, while the upper limit for underweight women (BMI $<18.5 \mathrm{~kg}$ / $\mathrm{m}^{2}$ ) is 18 kilos and for overweight or obese women (BMI $\geq 30 \mathrm{~kg} /$ $\mathrm{m}^{2}$ ) is 11.5 kilos or should not exceed 9 kilos, respectively [93].

\section{Effects of malnutrition and of excessive weight gain including obesity in pregnancy}

The short and long-term effects of maternal dietary behavior during pregnancy are currently being intensively studied. The consumption of large amounts of meat, sweetened beverages, sweetened or salty foods may potentially increase the risk of gestational hypertensive disorders (e.g. pre-eclampsia), or lead to premature delivery and small-for-gestational age (SGA) infants [4,94]. Two prospective population-based cohort studies have provided initial evidence of the long-term importance of pregnant women's diets on the development of their children, whereby unbalanced and unhealthy nutritional patterns of pregnant women (e.g. the consumption of large amounts of meat, sweetened beverages and salty snacks, low amounts of vegetables and fruit) were associated with later externalizing but not internalizing behavioral problems in their children $[95,96]$.

An inadequate dietary intake of micronutrients (e.g. iron or calcium deficiency) is associated with many medical complications [97]. On the other hand, the intake of micronutrients (i.e. taking vitamin, folic acid or iron supplements) in addition to the pregnant woman's regular intake of food can reduce the risk of neural tube defects, premature delivery, low birth weight and the risk of giving birth to a SGA baby [98]. A recent study showed that taking multiple micronutrients resulted in significantly superior outcomes compared to taking individual iron supplements with or without the addition of folic acid [99]. The late sequelae of a lack of micronutrients (primarily iron and iodine deficiency) in pregnancy can include impaired motor, cognitive and socialemotional development in affected children $[92,100]$.

Consequences of excessive maternal weight gain during pregnancy include birth complications (e.g. cesarean section) and 
postpartum maternal weight retention [93]. Excessive maternal weight gain can also affect fetal growth; consequences can include excessive fetal growth (LGA) or high birth weight [101]. Excessive weight gain can increase the subsequent risk of obesity for the child [102]. Women who were underweight before becoming pregnant or who did not gain sufficient weight during pregnancy have a higher risk of premature delivery and of giving birth to low birth weight or small-for-gestational-age children [101,103-105]. Other suggested long-term consequences of intrauterine malnutrition include postnatal catch-up growth, which has an associated increased risk of obesity but also of renal and cardiovascular disease in adulthood (e.g. arterial hypertension) $[4,106]$.

Maternal obesity which existed prior to the pregnancy has been investigated in many studies and is in itself a risk factor for perinatal complications. Marchi et al. [5] analyzed 22 reviews and meta-analyses on this issue published between 2007 and 2014 . Complications arising as a consequence of maternal obesity can include an increased risk of spontaneous miscarriage and malformation in early pregnancy (e.g. neural tube defects), gestational diabetes, gestational hypertension, pre-eclampsia, delivery by cesarean section, strong bleeding post partum, a higher risk of congenital anomalies, premature delivery, and a risk of LGA children [5]. Over the long term, children of mothers who were already overweight or obese during pregnancy are themselves at increased risk of becoming overweight or obese [103] and of developing hypertension and diabetes mellitus type II in later life [107]. In addition, there are initial indications pointing to a link between later cognitive impairments as well as externalizing and internalizing behavioral disorders and maternal obesity during pregnancy [108-109].

\section{Prevalence of malnutrition and excessive weight gain/obesity during pregnancy}

An inadequate intake of macro- and micronutrients is not just a significant health problem in low income countries [109]. According to recent meta-analyses, pregnant women in the USA, Canada, the United Kingdom, Japan, Australia and New Zealand are ingesting too much fat and saturated fatty acids [110] while the intake of folic acid, iron, iodine, and vitamin D supplements is too low and does not match national recommendations [111]. Moreover, there has been a significant increase in the number of overweight and obese women of child-bearing age worldwide [109]. The results of a perinatal survey in Germany showed that the percentage of pregnant women with a BMI of $30-40 \mathrm{~kg} / \mathrm{m}^{2}$ increased from $8.2 \%$ in 1995 to $13.0 \%$ in 2011 ; the percentage of women with a BMI $\geq 40 \mathrm{~kg} / \mathrm{m}^{2}$ even tripled to $1.8 \%$, while the percentage of underweight pregnant women $\left(\mathrm{BMI}<18.5 \mathrm{~kg} / \mathrm{m}^{2}\right.$ ) remained almost constant at $4 \%$ [33].

\section{Correlates of malnutrition and of excessive weight gain/obesity}

A search of the literature identified six systematic reviews (one which included a meta-analysis) which summarized potential predictors of malnutrition or excessive weight gain and obesity. In addition, a further search of the literature revealed 19 empirical individual studies ( $\mathrm{n}=5$ studies on dietary habits; $\mathrm{n}=4$ studies on the intake of micronutrients using the intake of folic acid supplements as an example; $\mathrm{n}=10$ studies on weight gain) published between 2005 and 2016 (cf. $\odot$ Table 2). The findings on dietary habits are presented as a first step below; in a second step the findings on the determinants of excessive weight gain and obesity are summarized below.

Dietary habits and quality are significantly affected by socio-demographic, socio-economic, socio-cultural, psychosocial and behavioral factors [112]. These factors are also important for dietary behavior during pregnancy. Studies have shown that malnutrition during pregnancy caused either by a frequent intake of fatty or sugar foods, an insufficient intake of healthy foods (e.g. fruit, vegetables, rice, fish) or an insufficient intake or lack of important micronutrients (e.g. folic acid) is commonly associated with a younger maternal age, immigrant status, a lower level of education, and being unemployed [77-78,81-82,84,86-87]. Single women or unmarried women in a steady relationship were also more prone not to eat health-consciously $[77,81,86]$. It is possible that a lack of integration in social relationships and a lack of social support encourages unhealthy eating habits $[37,77]$, but because of the limited number of studies it is not possible to draw clear conclusions on this point.

Attention is increasingly being focused on the associations between nutritional quality and high levels of stress or mental health problems. There are some initial indications that pregnant women who either have increased depression scores or who often suffer from stress tend to have a more unhealthy and unbalanced diet $[38,76,87]$. Wall et al. [87] reported that pregnant women with symptoms of depression tended to mainly eat a diet of junk food. Pregnant women with anxiety symptoms also appeared to have a tendency to eat less healthily (e.g. frequent consumption of sugary foods) $[77,81]$. Baskin et al. [38] interpreted the correlations between poor quality nutrition and mental health problems analogously to nicotine and alcohol consumption as constituting a type of coping mechanism; on the other hand the authors speculated that limited drive and low motivation to pay attention to one's own diet over the course of the entire pregnancy could play an important role.

Important health-related factors and behaviors which are associated with malnutrition include reduced physical activity [77], being overweight or obese pre-pregnancy $[81,84,85]$ and the continued consumption of nicotine or alcohol during pregnancy $[51,67,77,79,81,86,87]$. Pregnant smokers, for example, were more likely to live on a diet of fatty foods (e.g. junk food, chips) $[81,87]$ compared to pregnant non-smokers and also were much less likely to take folic acid supplements $[51,67,79,86]$. A diet lacking in micronutrients was more common among women who had already given birth to several children [78,79,82,86], while indications of an unhealthy and unbalanced diet were reported for both nullipara and multipara [77, 81, 84,87].

The possible determinants for excessive weight gain have often been investigated. Recent reviews have focused exclusively on the psychosocial predictors of excessive weight gain (cf. $\square$ Table 1). Ten empirical studies which examined the importance of socio-demographic, lifestyle-related and health-related factors published between 2005 and 2016 were also included in this review (cf. $\odot$ Table 2).

Socio-demographic risk factors (e.g. age of the expectant mother) are often discussed in the context of excessive weight gain in pregnancy; however, recent studies have not shown a consistent picture (cf. $\bigcirc$ Table 2). A much greater importance is attached to modifiable psychosocial and health-related factors $[39,40]$. For example, studies have shown a correlation between excessive weight gain in pregnancy and dissatisfaction with body image prior to becoming pregnant (e.g. a preference for being thin) or an inadequate perception of one's own bodyweight. There are 
also indications that women with excessive weight gain in pregnancy often also show symptoms of depression, which may be based on an association between dissatisfaction with their own body image and excessive weight gain in pregnancy [39]. Overweight and obese women were also more likely to have symptoms of depression during pregnancy and after the birth compared to normal-weight women [41]. Likewise, obese pregnant women appeared to have more symptoms of anxiety, although the overall findings on this point are less conclusive because of the limited number of studies and their lack of methodological quality [42].

Pre-pregnancy body mass index is the most important health-related risk factor for excessive weight gain during pregnancy and can easily be measured during a routine antenatal check-up. Overweight and obese women are particularly at risk of gaining too much weight during pregnancy [69-71,73-74, 83]. But an expectant mother who herself had a high birth weight [83] and the body mass index of the expectant father were also found to be associated with excessive weight gain during pregnancy [72]. In addition, continued nicotine consumption [72,74], quitting smoking, and lack of physical activities $[69,80,83]$ can also lead to excessive weight gain [83]. In contrast, regular physical activity during pregnancy can have a preventive effect on weight gain in pregnancy. Women who are physically active over a period of at least two hours per week are less likely to exceed the recommended guideline values for weight gain in pregnancy compared to women who are physically inactive [73-74]. The number of previous births can also affect the amount of weight gained in pregnancy. Women who have not previously given birth have a higher risk of excessive weight gain during pregnancy than multipara $[70,72-73,75,80]$. Conversely, multipara are more likely to be overweight or obese but less likely to have excessive weight gain in pregnancy $[72,75]$.

\section{Mental State}

In the last three decades, interest in investigating the effects of the pregnant woman's mental state on the pregnancy and birth as well as on the development of the fetal and child has increased [6]. A search of the literature has shown that the overwhelming majority of systematic reviews and meta-analyses have focused on the relationship between emotional disturbances, i.e. stress, depression or anxiety experienced by the expectant mother and the birth parameters of the child. This review will therefore also limit itself to looking only at these factors. It is basically assumed that chronic, excessively emotional stress experienced by the pregnant woman can lead to dysregulation of maternal and fetal hypothalamic-pituitary-adrenal axis activity [113]. In the placental barrier, the presence of $11 \beta$-hydroxysteroid dehydrogenase (11ß-HSD) type 2 , an enzyme that catalyzes the conversion of cortisol into biologically inactive cortisone, normally protects the fetus from excessive concentrations of cortisol [15]. However, it has been postulated that chronically increased levels of stress hormones could result in increased permeability through downregulation of the $11 \beta-H S D 2$ enzyme, allowing more cortisol to reach the fetal blood stream [113]. Prenatal exposure to excessively high levels of stress hormones can adversely affect the neural, immunological and behavioral development of the child, predisposing it to be more susceptible to psychological and physical disease(s) in later life [114].
Effects of stress, depression and anxiety in pregnancy

Pregnant women with depression and anxiety are more likely to suffer from pregnancy symptoms such as nausea and vomiting [115]. While Grigoriadis et al. [116] found no correlation between depression and pre-eclampsia, a more recent study [117] showed that depressive symptoms in pregnant women may be associated with an increased risk of pre-eclampsia. Moreover, a number of studies have noted that depressive and anxious pregnant women consulted their doctors more often and were more likely to require pain relief during the birth [115]. Other perinatal complications arising from mild to clinically relevant symptoms of prenatal maternal stress, depression or anxiety can include premature delivery and low birth weight of the child [118-120]. There were also indications that depression in the mother can predispose to intrauterine growth retardation [121-122]. If maternal mental health problems remain untreated, there is a risk that these symptoms will continue even after the birth of the child [123124 ], which can seriously limit the maternal care given to the infant and the interaction between mother and infant [125-127]. Children of mothers affected prenatally by stress and depression often have difficulties adapting to new and stressful events in early childhood, are more easily agitated and more likely to show deficits in regulating their attention, behavior and emotions [128-129]. There is evidence that maternal psychological distress can result in developmental delays which affect the infant's motor skills and cognitive development [130-132]. In the long term, maternal depression and anxiety during pregnancy can increase the child's risk of developing emotional and behavioral disorders [129]. Prenatal maternal depressive disorders are a significant risk factor for later aggressive behavior on the part of the child [133].

\section{Prevalence of stress, depression and anxiety} in pregnancy

Because of the wide-ranging physiological, psychological and social changes experienced by the expectant mother, the time of pregnancy and birth are a stage in which women may be much more vulnerable to stress and mental health problems $[15,123]$. A survey of 1522 pregnant women showed, for example, that up to $78 \%$ of women had slight to moderate stress and $6 \%$ of pregnant women experienced high levels of stress during pregnancy [134]. Uncertainty, anxiety and worries about the health of the unborn child or the forthcoming birth are part of the normal adjustment processes of women to pregnancy and their future maternal role, but the symptoms become clinically relevant if they continue to be experienced inordinately powerfully over a longer period of time and result in a significant functional impairment of the pregnant woman [123]. Depression and anxiety are the most common psychological disorders found in pregnancy. Up to $18.4 \%$ of women develop depressive symptoms during pregnancy, and around $12.7 \%$ of pregnant women suffer from major depression requiring treatment [135]. The figures for the prevalence of anxiety disorders in pregnancy range from 4.4 to $39 \%$ [136]. Pregnancy-specific anxiety is very common; approximately $34 \%$ of pregnant women are anxious about the impending birth, $32.4 \%$ of women are afraid of perineal tears or an episiotomy, and $26.8 \%$ are scared of the labor pains [137].

\section{Correlates of depression and anxiety in pregnancy}

The literature search located four systematic reviews which looked at potential risk factors in various areas (e.g. socio-demographic factors, psychological/psychiatric or obstetrical/preg- 
nancy-specific factors) for prenatal symptoms of depression and anxiety and maternal psychological disorders during pregnancy which required treatment (cf. O Table 1). Four more systematic reviews and/or meta-analyses published between 2013 and 2016 were identified which investigated specific individual risk factors (e.g. domestic violence, unintended pregnancy) in connection with maternal depression and anxiety during pregnancy. No further searches for recent empirical individual studies were carried out.

Frequently investigated socio-demographic and economic factors which can contribute to the development of depression and anxiety disorders during pregnancy include a young maternal age, being single, a low income, and a low level of education $[45,46$, 48-49]. Conclusive findings were most likely to be correlated to prenatal anxiety disorders [49], while the association between depressive symptoms and socio-demographic or economic factors were potentially affected by the country in which the data were collected $[45-46,48]$. No conclusive findings have yet been identified regarding the immigration status or ethnicity of pregnant women $[46,48]$. Consistent social risk factors for symptoms of depression or anxiety in pregnancy were lack of or inadequate social support by the woman's partner and conflicts with their partner $[46,48-50]$. The emotional support and practical help given by the partner or by close friends or relatives plays an important role in adjusting to motherhood, managing events and coping with difficulties which can develop in the context of pregnancy and the birth of a child [45]. Frequent conflicts in their intimate relationship or dissatisfaction with their partner may be perceived as distressing by the pregnant woman and can significantly affect her adjustment to motherhood. Appreciative and sympathetic support by the partner and emotional closeness are an important resource during the transition to parenthood which can reduce the risk of perinatal depression or anxiety [50].

Previous psychiatric illnesses of the pregnant woman - both illnesses experienced during prior pregnancies or at other stages in the woman's life - play an important role for the development of depression and anxiety in pregnancy $[45-46,49]$. But there are also indications that the majority of affected women never had a previous mental illness [45]. Depression and anxiety often occur as co-morbidities, whereby pronounced symptoms of anxiety in the pregnant woman can predispose her to go on to develop depression [45]. Critical life events (e.g. the loss of a close relative or friend) and the perception of high levels of stress by the pregnant woman can increase the risk of prenatal depression and anxiety $[45,48]$. Affected women may often suffer from feelings of guilt or worry about the health and development of their unborn child. Women who have experienced various forms of abuse (e.g. abuse in childhood, experience of intimate partner violence) are a group with a particularly high risk of depression or anxiety during pregnancy [44]. Between 1.8 and $31.7 \%$ of pregnant women are victims of violence, although the number of unrecorded cases is likely to be far higher [138]. Women with symptoms of depression or anxiety have a three to five times higher risk of experiencing partner violence [47]. It is generally assumed that physically violent conflicts in a relationship often persist after the birth of a child and these conflicts, in their turn, have a lasting impact on the development of the child [139]. Women who have experienced violence tend to have a more negative attitude toward pregnancy and more difficulty in bonding with their unborn child than pregnant women who have not experienced violence [140]. After the birth women with a prior history of experiencing violence during pregnancy may have difficulty in establishing a close relationship with their infant which can increase the potential risk of child neglect and abuse by the mother [141], particularly if additional psychosocial risk factors are also present [142]. The previous sections have demonstrated that women who continued to smoke during pregnancy, who had an unhealthy or unbalanced diet, or who gained an excessive amount of weight, usually also had symptoms of depression and/or anxiety [38,39,41]. But it is still unclear whether mental health problems and stress affect the health behavior or pregnant women or whether health-damaging behaviors lead to depression or anxiety [45]. As manifestations of depressive or anxious symptoms were not usually collected prior to pregnancy, the findings described here must be interpreted, for the time being, as co-morbidities [42]. Obstetrical and pregnancy-specific factors can also play an important role in the development of depression and anxiety. Studies have shown that an unintended pregnancy can predispose the pregnant women to develop depression or anxiety (cf. @ Table 1). According to the findings of Abajour et al. [43], up to $21 \%$ of women with an unwanted or unplanned pregnancy develop depression during pregnancy or around the time of the birth. A sudden, unexpected perinatal loss of a child (e.g. due to miscarriage or stillbirth) can trigger complicated grief reactions as well as promoting the development of mental disorders [143]. Previous pregnancy loss (e.g. miscarriage and stillbirth), neonatal death, and current or previous pregnancy complications can lead to increased anxiety and depression scores, culminating in anxiety and/or depression disorders requiring treatment in subsequent pregnancies $[45,49]$. An interval of less than six months between the loss of a pregnancy and a new pregnancy may be particularly important in this context [45].

\section{Conclusions for Clinical Practice \\ $\nabla$}

Pregnancy and the birth of a child are an especially good time for preventive healthcare measures (e.g. weight normalization, smoking cessation, stress reduction, nutritional counselling) [1]. Pregnant women usually are very aware of the potential risks for their unborn child [144] and are correspondingly open to changes which would positively affect the health of their unborn child. The majority of pregnant women are prepared to reduce the amount of nicotine and/or alcohol they consume or even to stop smoking or drinking completely and are prepared to eat a healthy diet [145]. Nevertheless, a considerable percentage of pregnant women continue to display health-damaging behaviors, although the data on the incidence of this risky behavior, particularly with respect to nicotine and alcohol consumption in pregnancy, varies considerably depending on the country where the data was collected (cf. $\odot$ Table 2 ). It is generally assumed that maternal nicotine and alcohol consumption during pregnancy is underreported [146]. There are many reasons for this [146,147]. The experience of emotional stress is also very common among pregnant women. Mental health problems usually go undetected as the symptoms of depression and anxiety (e.g. increased fatigue, exhaustion, lack of energy, worries) cannot be clearly differentiated from pregnancy-specific problems. A knowledge of the predisposing factors could therefore provide early indications, making it easier for gynecologists to identify affected women early on during routine antenatal check-ups and take appropriate steps. The main aim of this article was therefore to give an overview of the potential factors predisposing to health-damaging behaviors and mental health problems in pregnancy. 
Table 3 Summary of the findings on factors affecting health-damaging behaviors and mental health problems during pregnancy.

\begin{tabular}{|c|c|c|c|c|c|}
\hline \multicolumn{3}{|c|}{ Health-damaging behaviors } & \multicolumn{3}{|c|}{ Mental disorders } \\
\hline $\begin{array}{l}\text { Nicotine con- } \\
\text { sumption }\end{array}$ & $\begin{array}{l}\text { Alcohol con- } \\
\text { sumption }\end{array}$ & Malnutrition & $\begin{array}{l}\text { Excessive weight } \\
\text { gain/obesity }\end{array}$ & Depression & Anxiety disorder \\
\hline \multicolumn{6}{|l|}{ Social factors } \\
\hline $\begin{array}{l}\text { younger maternal } \\
\text { age (aged less than } \\
25 \text { years) } \\
\text { [32,61,62] } \\
\text { lower socio- } \\
\text { economic status/ } \\
\text { level of education/ } \\
\text { income [32, 35, 51, } \\
\text { 56-58,61,65,68] } \\
\text { single [57,61,67] } \\
\text { characteristics of } \\
\text { relationship with } \\
\text { intimate partner } \\
\text { (lack of support by } \\
\text { partner [37], rela- } \\
\text { tionship conflicts } \\
\text { [54], violence [65]) } \\
\text { partner smokes [35] }\end{array}$ & $\begin{array}{l}\text { higher maternal age } \\
\text { (aged more than } \\
30 \text { years) } \\
{[36,61,63,66]} \\
\text { ' high level of educa- } \\
\text { tion }[60,61] \\
\text { characteristics of } \\
\text { relationship with } \\
\text { intimate partner } \\
\text { (partner is violent } \\
\text { [65]) }\end{array}$ & $\begin{array}{l}\text { younger maternal } \\
\text { age (aged less than } \\
25 \text { years) } \\
{[78,81,84,86,87]} \\
\text { immigrant back- } \\
\text { ground }[77,81,86]\end{array}$ & $\begin{array}{l}\text { inconsistent data } \\
\text { with regard to age } \\
{[71,74,80,83]} \\
\text { single } \\
{[74,77,81,86]} \\
\text { lack of social } \\
\text { support }[39]\end{array}$ & $\begin{array}{l}\text { inconsistent data } \\
\text { with regard to age } \\
{[45,46,48]} \\
\text { single }[46] \\
\text { characteristics of } \\
\text { relationship with } \\
\text { intimate partner } \\
\text { (lack of support by } \\
\text { partner }[45,46,50] \text {, } \\
\text { conflicts }[50], \text { vio- } \\
\text { lence }[45,47,48]) \\
\text { partner smokes/ } \\
\text { drinks }[50] \\
\text { lack of social } \\
\text { support }[\mathbf{4 6 , 4 8 , 5 0 ]}\end{array}$ & $\begin{array}{l}\text { young maternal age } \\
\text { (aged less than } \\
25 \text { years) }[49] \\
\text { single }[46,49] \\
\text { characteristics of } \\
\text { relationship with } \\
\text { intimate partner } \\
\text { (lack of support by } \\
\text { partner }[45,46,50] \text {, } \\
\text { conflicts }[49,50] \text {, } \\
\text { violence }[45,47] \text { ) } \\
\text { lack of social } \\
\text { support }[46,49,50]\end{array}$ \\
\hline \multicolumn{6}{|l|}{ Psychological factors } \\
\hline $\begin{array}{l}\text { prenatal depres- } \\
\text { sion/anxiety } \\
{[51,53,54,59,68]} \\
\text { - } \\
\text { stress/critical life } \\
\text { events }[54]\end{array}$ & $\begin{array}{l}\text { experience of } \\
\text { abuse/ill-treatment } \\
\text { [36] } \\
\text { stress/critical life } \\
\text { events [52] }\end{array}$ & $\begin{array}{l}\text { prenatal depres- } \\
\text { sion/anxiety } \\
{[38,81,87]} \\
\text { stress/critical life } \\
\text { events }[38,76]\end{array}$ & $\begin{array}{l}\text { prenatal depres- } \\
\text { sion/anxiety } \\
{[\mathbf{4 1 , 4 2}]}\end{array}$ & $\begin{array}{l}\text { stress/critical life } \\
\text { events }[\mathbf{4 5}, \mathbf{4 8}] \\
\text { experience of } \\
\text { ill-treatment/ } \\
\text { abuse }[\mathbf{4 4 , 4 7 ]} \\
\text { prior psychiatric } \\
\text { disorder }[45,46,49]\end{array}$ & $\begin{array}{l}\text { stress/critical life } \\
\text { events [45] } \\
\text { experience of } \\
\text { ill-treatment/ } \\
\text { abuse [47] } \\
\text { prior psychiatric } \\
\text { disorder }[\mathbf{4 5}, \mathbf{4 6 , 4 9 ]}\end{array}$ \\
\hline \multicolumn{6}{|c|}{ Health-related factors prior to the pregnancy } \\
\hline $\begin{array}{l}\text { frequency and } \\
\text { amount of nicotine } \\
\text { consumption } \\
{[35,54,65]} \\
\text { consumption } \\
\text { of drugs [62] }\end{array}$ & $\begin{array}{l}\text { frequency and } \\
\text { amount of alcohol } \\
\text { consumed } \\
{[36,55,60,66]} \\
\text { - consumption } \\
\text { of drugs } \\
\text { - }[63]\end{array}$ & $\begin{array}{l}\text { overweight/obese } \\
{[81,84,85]}\end{array}$ & $\begin{array}{r}\text { overweight/obese } \\
{[69-71,73,74,83]}\end{array}$ & & \\
\hline \multicolumn{6}{|c|}{ Health-related factors during pregnancy } \\
\hline $\begin{array}{l}\text { alcohol consump- } \\
\text { tion }[51,58,62]\end{array}$ & $\begin{array}{l}\text { nicotine consump- } \\
\text { tion }[36,55,63-65]\end{array}$ & $\begin{array}{l}\text { nicotine consump- } \\
\text { tion }[51,67,72,74 \text {, } \\
77,79,81,86,87] \\
\text { alcohol consump- } \\
\text { tion }[86,87]\end{array}$ & $\begin{array}{l}\text { nicotine consump- } \\
\text { tion }[72,74] \\
\text { lack of exercise } \\
\\
{[69,80,83]}\end{array}$ & $\begin{array}{l}\text { nicotine consump- } \\
\text { tion [45] }\end{array}$ & \\
\hline \multicolumn{6}{|c|}{ Pregnancy-specific factors } \\
\hline $\begin{array}{l}\text { high number of } \\
\text { previous births } \\
\text { (more than 2) } \\
{[32,35,56,57,65 \text {, }} \\
67] \\
\text { - unplanned preg- } \\
\text { nancy }[51,62,67] \\
\text { inadequate prenatal } \\
\text { care }[32,35,61]\end{array}$ & $\begin{array}{l}\text { high number of } \\
\text { previous births } \\
\text { (more than 2) } \\
{[36,61,65]} \\
\text { unplanned preg- } \\
\text { nancy }[55,60] \\
\text { pregnancy } \\
\text { complications [51] }\end{array}$ & $\begin{array}{l}\text { high number of } \\
\text { previous births } \\
\text { (more than 2) } \\
{[78,79,81,82,84 \text {, }} \\
86] \\
\text { unplanned preg- } \\
\text { nancy [86] }\end{array}$ & $\begin{array}{l}\text { no prior births } \\
{[70,72,73,75,80]}\end{array}$ & $\begin{array}{l}\text { unintended preg- } \\
\text { nancy }[\mathbf{4 3 , 4 5 , 4 6 ]} \\
\text { previous pregnancy } \\
\text { complications/ } \\
\text { pregnancy loss }[45]\end{array}$ & $\begin{array}{l}\text { unintended preg- } \\
\text { nancy }[\mathbf{4 5 , 4 6 , 4 9 ]} \\
\text { previous pregnancy } \\
\text { complications/ } \\
\text { pregnancy loss } \\
{[\mathbf{4 5 , 4 9 ]}}\end{array}$ \\
\hline
\end{tabular}

Note: Figures in square brackets refer to the sources. Systematic reviews and meta-analyses are indicated in bold.

The review of the literature showed that the continued consumption of substances such as alcohol or nicotine, malnutrition, excessive weight gain culminating in obesity, and impaired mental health all occur more frequently when certain psychosocial, health-related or pregnancy-specific features are present in the expectant mother, most of which existed already prior to the pregnancy. It also became clear that there are complex interactions between individual health-damaging behaviors as well as links to mental health problems, although the direction of these interactions is not yet clear. The results of this review are summarized in $\bullet$ Table 3.

Many of the factors described as affecting the health-related behavior or mental condition of pregnant women can easily be identified during routine antenatal check-ups or even prior to the pregnancy. The focus of antenatal care should therefore not be exclusively on assessing potential medical risks for the preg- 
nant women and her unborn child. Instead, gynecologists should take enough time when taking the medical history of their pregnant patients to record social and psychological factors in addition to medical risks and also to frankly address the pregnant woman's consumption of alcohol and nicotine and her diet. Antenatal care appointments should also be used to regularly broach the topic of possible psychosocial and pregnancy-related problems during the course of the pregnancy [148].

This article has provided a broad overview of possible factors which can predispose to health-damaging behaviors and mental health problems during pregnancy. As this article is not a systematic review and the focus is not on a specific individual risk factor in pregnancy it was impossible to include all relevant empirical studies dedicated to this topic. It is possible that further important predictors such as the characteristics of the woman's partner (e.g. psychiatric illness, consumer behavior or unemployment) or the pregnant woman's health-related attitudes, convictions or intention to modify her behavior have been overlooked.

\section{Conflict of Interest}

None.

\section{References}

1 Meinlschmidt G, Tegethoff M. How life before birth affects human health and what can we do about it. Eur Psychol 2015; 20: 85-89

2 Nigg JT. Where do epigenetics and developmental origins take the field of developmental psychopathology? J Abnorm Child Psychol 2016; 44: 405-419

3 Spohr HL. Teratogene Effekte von Nikotin, Drogen und Alkohol. Gynakologe 2005; 38: 25-32

4 Cetin I, Laoreti A. The importance of maternal nutrition for health. J Pediatr Neonatal I 2014; 4: e04040220

5 Marchi J, Berg M, Dencker A et al. Risks associated with obesity in pregnancy, for the mother and baby: a systematic review of reviews. Obes Rev 2015; 16: 621-638

6 Dunkel-Schetter C, Tanner L. Anxiety, depression and stress in pregnancy: implications for mother, children, research and practice. Curr Opin Psychiatry 2012; 25: 141-148

7 Huizink AC. Prenatal maternal substance use and offspring outcomes: overview of recent findings and possible interventions. Eur Psychol 2015; 20: 90-101

8 Schneider H, Husslein PW, Schneider KT, Hrsg. Die Geburtshilfe. Berlin: Springer; 2011: 617-632

9 Schmidt MH, Petermann F, Schipper M. Epigenetik - Revolution der Entwicklungspsychopathologie? Kindh Entwickl 2012; 21: 245-253

10 Monk C, Spicer J, Champagne FA. Linking prenatal maternal adversity to developmental outcomes in infants: The role of epigenetic pathways. Dev Psychopathol 2012; 24: 1361-1376

11 Lampert T, Richter M, Schneider $S$ et al. Soziale Ungleichheit und Gesundheit. Stand und Perspektiven der sozialepidemiologischen Forschung in Deutschland. Bundesgesundheitsbl 2016; 59: 153-165

12 De Graaf JP, Steegers EA, Bonsel GJ. Inequalities in perinatal and maternal health. Curr Opin Obstet Gyn 2013; 25: 98-108

13 Goeckenjan M, Ramsauer B, Hänel M et al. Soziales Risiko - geburtshilfliches Risiko? Gynäkologe 2009; 42: 102-110

14 Pillas D, Marmot $M$, Naicker $K$ et al. Social inequalities in early childhood health and development: a European-wide systematic review. Pediatr Res 2014; 76: 418-424

15 La Marca-Ghaemmaghami P, Ehlert $U$. Stress during pregnancy. Experienced stress, stress hormones, and protective factors. Eur Psychol 2015; 20: 102-119

16 Mund M, Louwen F, Klingelhoefer D et al. Smoking and pregnancy - a review on the first major environmental risk factor of the unborn. Int J Environ Res Public Health 2013; 10: 6485-6499

17 Wessels C, Winterer G. Nikotin und Gehirnentwicklung. Nervenarzt 2008; 79: 7-16

18 Hösli I, Zanetti-Daellenbach R, Holzgreve W et al. Rauchen während der Schwangerschaft. Geburtsh Frauenheilk 2008; 68: 141-146
19 Marufu TC, Ahankari A, Coleman T et al. Maternal smoking and the risk of still birth: systematic review and meta-analysis. BMC Public Health 2015; 15: 239

20 Pineles BL, Park E, Samet JM. Systematic review and meta-analysis of miscarriage and maternal exposure to tobacco smoke during pregnancy. Am J Epidemiol 2014; 179: 807-823

21 Koch S, Vilser C, Groß W et al. Rauchen während der Schwangerschaft Risiko für intrauterine Wachstumsrestriktion und bleibende Kleinwüchsigkeit. Z Geburtshilfe Neonatol 2012; 216: 77-81

22 Hackshaw A, Rodeck C, Boniface S. Maternal smoking in pregnancy and birth defects: a systematic review based on 173687 malformed cases and 11.7 million controls. Hum Reprod Update 2011; 17: 589-604

23 Zhou S, Rosenthal DG, Sherman S et al. Physical, behavioral, and cognitive effects of prenatal tobacco and postnatal secondhand smoke exposure. Curr Probl Pediatr Adolesc Health Care 2014; 44: 219-241

24 Latimer K, Wilson P, Kemp J et al. Disruptive behaviour disorders: a systematic review of environmental antenatal and early years risk factors. Child Care Health Dev 2012; 38: 611-628

25 Tiesler CM, Heinrich J. Prenatal nicotine exposure and child behavioural problems. Eur Child Adolesc Psychiatry 2014; 23: 913-929

26 Clifford A, Lang L, Chen R. Effects of maternal cigarette smoking during pregnancy on cognitive parameters of children and young adults: a literature review. Neurotoxicol Teratol 2012; 34: 560-570

27 Dörrie N, Föcker M, Freunscht I et al. Fetal alcohol spectrum disorders. Eur Child Adolesc Psychiatry 2014; 23: 863-875

28 Patra J, Bakker $R$, Irving $H$ et al. Dose-response relationship between alcohol consumption before and during pregnancy and the risks of low birthweight, preterm birth and small for gestational age (SGA) - a systematic review and meta-analyses. BJOG 2011; 18: 1411-1421

29 Flak AL, Su S, Bertrand J et al. The association of mild, moderate and binge prenatal alcohol exposure and child neuropsychological outcomes: a meta-analysis. Alcohol Clin Exp Res 2014; 38: 214-226

30 Alvik A, Aalen OO, Lindemann R. Early fetal binge alcohol exposure predicts high behavioral symptom scores in 5.5-year-old children. Alcohol Clin Exp Res 2013; 37: 1954-1962

31 Niclasen J, Andersen AM, Strandberg-Larsen $K$ et al. Is alcohol binge drinking in early and late pregnancy associated with behavioural and emotional development at age 7 years? Eur Child Adolesc Psychiatry 2014; $23: 1175-1180$

32 Schneider S, Maul H, Freerksen $N$ et al. Who smokes during pregnancy? An analysis of the German Perinatal Quality Survey 2005. Public Health 2008; 122: 1210-1216

33 Scholz R, Voigt M, Schneider $K$ et al. Analysis of the German Perinatal Survey of the years 2007-2011 and comparison with data from 19951997: maternal characteristics. Geburtsh Frauenheilk 2013; 73: $1247-$ 1251

34 Pfinder M, Feldmann R, Liebig S. Alcohol during pregnancy from 1985 to 2005: prevalence and high risk profile. Sucht 2013; 59: 165-173

35 Schneider S, Huy C, Schütz J et al. Smoking cessation during pregnancy: a systematic literature review. Drug Alcohol Rev 2010; 29: 81-90

36 Skagerstróm J, Chang G, Nilsen P. Predictors of drinking during pregnancy: a systematic review. J Womens Health 2011; 20: 901-913

37 Ulrich F, Petermann F. Zur Bedeutung von sozialer Unterstützung während der Schwangerschaft. Psychol Rundsch 2014; 65: 57-74

38 Baskin R, Hill B, Jacka FN et al. The association between diet quality and mental health during the perinatal period. A systematic review. Appetite 2015; 91: 41-47

39 Hartley E, McPhie S, Skouteris H et al. Psychosocial risk factors for excessive gestational weight gain: A systematic review. Women Birth 2015; 28: e99-e109

40 Kapadia MZ, Gaston A, Van Blyderveen S et al. Psychological antecedents of excess gestational weight gain: a systematic review. BMC Pregnancy Childbirth 2015; 15: 107

41 Molyneaux E, Poston L, Ashurst-Williams S et al. Obesity and mental disorders during pregnancy and postpartum: a systematic review and meta-analysis. Obstet Gynecol 2014; 123: 857-867

$42 \mathrm{Nagl} M$, Linde K, Stepan $\mathrm{H}$ et al. Obesity and anxiety during pregnancy and postpartum: a systematic review. J Affect Disord 2015; 186: 293305

43 Abajobir AA, Maravilla JC, Alati $R$ et al. A systematic review and metaanalysis of the association between unintended pregnancy and perinatal depression. J Affect Disord 2016; 192: 56-63

44 Alvarez-Segura M, Garcia-Esteve L, Torres A et al. Are women with a history of abuse more vulnerable to perinatal depressive symptoms? A systematic review. Arch Womens Ment Health 2014; 17: 343-357 
45 Biaggi A, Conroy S, Pawlby S et al. Identifying the women at risk of antenatal anxiety and depression: a systematic review. J Affect Disord 2016; 191: 62-77

46 Fisher J, Cabral de Mello M, Patel Vet al. Prevalence and determinants of common perinatal mental disorders in women in low- and lower-middle-income countries: a systematic review. Bull World Health Organ 2012; 90: 139-149

47 Howard LM, Oram S, Galley $H$ et al. Domestic violence and perinatal mental disorders: a systematic review and meta-analysis. PLoS Med 2013; 10: e1001452

48 Lancaster CA, Gold KJ, Flynn HA et al. Risk factors for depressive symptoms during pregnancy: a systematic review. Am J Obstet Gynecol 2010; 202: 5-14

49 Leach LS, Poyser C, Fairweather-Schmidt K. Maternal perinatal anxiety: a review of prevalence and correlates. Clinical Psychologist 2015; DOI: $10.1111 / \mathrm{cp} .12058$

50 Pilkington PD, Milne LC, Cairns KE et al. Modifiable partner factors associated with perinatal depression and anxiety: a systematic review and meta-analysis. J Affect Disord 2015; 178: 165-180

51 Baron R, Manniën J, de Jonge A et al. Socio-demographic and lifestylerelated characteristics associated with self-reported any, daily and occasional smoking during pregnancy. PLoS One 2013; 8: e74197

52 Beijers C, Ormel J, Meijer JL et al. Stressful events and continued smoking and continued alcohol consumption during mid-pregnancy. PLoS One 2014; 9: e86359

53 De Wilde KS, Trommelmans LC, Laeven HH et al. Smoking patterns, depression, and sociodemographic variables among Flemish women during pregnancy and the postpartum period. Nurs Res 2013; 62: 394404

54 Hauge LJ, Torgersen L, Vollrath M. Associations between maternal stress and smoking: findings from a population-based prospective cohort study. Addiction 2012; 107: 1168-1173

55 Iversen ML, Sorensen NO, Broberg $L$ et al. Alcohol consumption and binge drinking in early pregnancy. A cross-sectional study with data from the Copenhagen Pregnancy Cohort. BMC Pregnancy Childbirth 2015; 15: 327

56 Kale PL, Fonseca SC, da Silva KS et al. Smoking prevalence, reduction, and cessation during pregnancy and associated factors: a cross-sectional study in public maternities, Rio de Janeiro, Brazil. BMC Public Health 2015; 15: 406

57 Kharkova OA, Krettek A, Grjibovski AM et al. Prevalence of smoking before and during pregnancy and changes in this habit during pregnancy in Northwest Russia: a Murmansk county birth registry study. Reprod Health 2016; 13: 18

58 Masho SB, Bishop DL, Keyser-Marcus L et al. Least explored factors associated with prenatal smoking. Matern Child Health J 2013; 17: 11671174

59 Maxson PJ, Edwards SE, Ingram A et al. Psychosocial differences between smokers and non-smokers during pregnancy. Addict Behav 2012; 37: 153-159

60 McDonald SW, Hicks M, Rasmussen C et al. Characteristics of woman who consume alcohol before and after pregnancy recognition in a Canadian Sample: a prospective cohort study. Alcohol Clin Exp Res 2014; 38: 3008-3016

61 Melchior M, Chollet A, Glangeaud-Freudenthal N et al. Tobacco and alcohol use in pregnancy in France: the role of migrant status. The nationally representative ELFE study. Addict Behav 2015; 51: 65-71

62 Murphy DJ, Mullally A, Cleary BJ et al. Behavioural change in relation to alcohol exposure in early pregnancy and impact on perinatal outcomes - a prospective cohort study. BMC Pregnancy Childbirth 2013; 13: 8

63 Murphy DJ, Dunney C, Mullally A et al. Population-based study of smoking behaviour throughout pregnancy and adverse perinatal outcomes. Int J Environ Res Public Health 2013; 10: 3855-3867

64 O'Keeffe LM, Kearney PM, McCarthy FP et al. Prevalence and predictors of alcohol use during pregnancy: findings from international multicentre cohort studies. BMJ 2015; 5: e006323

65 Powers JR, McDermott LJ, Loxton DJ et al. A prospective study of prevalence and predictors of concurrent alcohol and tobacco use during pregnancy. Matern Child Health J 2013; 17: 76-84

66 Skagerström J, Alehagen S, Häggström-Nordin E et al. Prevalence of alcohol use before and during pregnancy and predictors of drinking during pregnancy: a cross sectional study in Sweden. BMC Public Health 2013; $13: 780$
67 Smedberg J, Lupattelli A, Mårdby AC et al. Characteristics of women who continue smoking during pregnancy: a cross-sectional study of pregnant women and new mothers in 15 European countries. BMC Pregnancy Childbirth 2014; 14: 213

68 Smedberg J, Lupattelli A, Mårdby AC et al. The relationship between maternal depression and smoking cessation during pregnancy-a crosssectional study of pregnant women from 15 European countries. Arch Womens Ment Health 2015; 18: 73-84

69 Althuizen E, van Poppel MN, Seidell JC et al. Correlates of absolute and excessive weight gain during pregnancy. J Womens Health (Larchmt) 2009; 18: 1559-1566

70 Brawarsky P, Stotland NE, Jackson RA et al. Pre-pregnancy and pregnancy-related factors and the risk of excessive or inadequate gestational weight gain. Int J Gynaecol Obstet 2005; 91: 125-131

71 Chasan-Taber L, Schmidt MD, Pekow P et al. Predictors of excessive and inadequate gestational weight gain in Hispanic women. Obesity 2008; 16: 1657-1666

72 Gaillard R, Durmus B, Hofman A et al. Risk factors and outcomes of maternal obesity and excessive weight gain during pregnancy. Obesity 2013; 21: 1046-1055

73 Herring SJ, Nelson DB, Davey A et al. Determinants of excessive gestational weight gain in urban, low-income women. Womens Health Issues 2012; 22: e439-e446

74 Kraschnewski JL, Chuang CH, Downs DS et al. Association of prenatal physical activity and gestational weight gain: results from the first baby study. Womens Health Issues 2013; 23: e233-e238

75 Lan-Pidhainy X, Nohr EA, Rasmussen KM. Comparison of gestational weight gain-related pregnancy outcomes in American primiparous and multiparous women. Am J Clin Nutr 2013; 97: 1100-1106

76 Lobel M, Cannella DL, Graham JE et al. Pregnancy-specific stress, prenatal health behaviors, and birth outcomes. Health Psychol 2008; 27: 604-615

77 Nash DM, Gilliland JA, Evers SE et al. Determinants of diet quality in pregnancy: sociodemographic, pregnancy-specific, and food environment influences. J Nutr Educ Behav 2013; 45: 627-634

78 Nilsen RM, Leoncini E, Gastaldi P et al. Prevalence and determinants of preconception folic acid use: an Italian multicenter survey. J Pediatr 2016; $42: 65$

79 Nilsen RM, Vollset SE, Gjessing HK et al. Patterns and predictors of folic acid supplement use among pregnant women: the Norwegian Mother and Child Cohort Study. Am J Clin Nutr 2006; 84: 1134-1141

80 Nohr EA, Vaeth M, Baker JL et al. Combined associations of prepregnancy body mass index and gestational weight gain with the outcome of pregnancy. Am J Clin Nutr 2008; 87: 1750-1759

81 Northstone N, Emmett P, Rogers I. Dietary patterns in pregnancy and associations with socio-demographic and lifestyle factors. Eur J Clin Nutr 2008; 62: 471-479

82 Pouchieu C, Lévy R, Faure C et al. Socioeconomic, lifestyle and dietary factors associated with dietary supplement use during pregnancy. PLoS One 2013; 8: e70733

83 Restall A, Taylor RS, Thompson JM et al. Risk factors for excessive gestational weight gain in a healthy, nulliparous cohort. J Obes 2014; 2014: 148391

84 Rifas-Shiman SL, Rich-Edwards JW, Kleinman KP et al. Dietary quality during pregnancy varies by maternal characteristics in project viva: a US cohort. J Am Diet Assoc 2009; 109: 1004-1011

85 Shin D, Lee KW, Song WO. Pre-pregnancy weight status is associated with diet quality and nutritional biomarkers during pregnancy. Nutrients 2016; 8: 162

86 Timmermans S, Jaddoe VW, Mackenbach JP et al. Determinants of folic acid use in early pregnancy in a multi-ethnic urban population in The Netherlands: the Generation R Study. Prev Med 2008; 47: 427-432

87 Wall CR, Gammon CS, Bandara DK et al. Dietary patterns in pregnancy in New Zealand-influence of maternal socio-demographic, health and lifestyle factors. Nutrients 2016; 8: 300

88 Ditzen B, Heinrichs M. Psychobiology of social support: the social dimension of stress buffering. Restor Neurol Neurosci 2014; 32: 149-162

89 Boucher J, Konkle AT. Understanding inequalities of maternal smokingbridging the gap with adapted intervention strategies. Int J Environ Res Public Health 2016; 13: E282

90 Berti C, Cetin I, Agostoni C et al. Pregnancy and infants' outcome: nutritional and metabolic implications. Crit Rev Food Sci 2016; 56: 82-91

91 Koletzko B, Bauer CP, Bung P et al. German National Consensus Recommendations on Nutrition and Lifestyle in Pregnancy by the 'Healthy Start - Young Family Network'. Ann Nutr Metab 2013; 63: 311-322 
92 Prado EL, Dewey KG. Nutrition and brain development in early life. Nutr Rev 2014; 72: 267-284

93 Rasmussen KM, Catalano PM, Yaktine AL. New guidelines for weight gain during pregnancy: what obstetrician/gynecologists should know. Curr Opin Obstet Gynecol 2009; 21: 521-526

94 Chen X, Zhao D, Mao Xet al. Maternal dietary patterns and pregnancy outcome. Nutrients 2016; 8: E351

95 Jacka FN, Ystrom E, Brantsaeter AL et al. Maternal and early postnatal nutrition and mental health of offspring by age 5 years: a prospective cohort study. J Am Acad Child Psy 2013; 52: 1038-1047

96 Steenweg-de Graaff J, Tiemeier R, Steegers-Theunissen P et al. Maternal dietary patterns during pregnancy and child internalising and externalising problems: the Generation R Study. Clin Nutr 2014; 33: 115121

97 Black RE, Victora CG, Walker SP et al. Maternal and child undernutrition and overweight in low-income and middle-income countries. Lancet 2013; 382: 427-451

98 Ramakrishnan U, Grant F, Goldenberg T et al. Effect of women's nutrition before and during early pregnancy on maternal and infant outcomes: a systematic review. Paediatr Perinat Ep 2012; 26 (Suppl. 1): 285-301

99 Haider BA, Bhutta ZA. Multiple-micronutrient supplementation for women during pregnancy. Cochrane Database Syst Rev 2012; (11): CD004905

100 Nyaradi A, Li JH, Hickling S et al. The role of nutrition in children's neurocognitive development, from pregnancy through childhood. Front Hum Neurosci 2013; 7: 97

101 Siega-Riz AM, Viswanathan M, Moos MK et al. A systematic review of outcomes of maternal weight gain according to the Institute of Medicine recommendations: birthweight, fetal growth, and postpartum weight retention. Am J Obstet Gynecol 2009; 339: e1-e14

102 Nehring I, Lehmann S, Kries R. Gestational weight gain in accordance to the IOM/NRC criteria and the risk for childhood overweight: a meta-analysis. Pediatr Obes 2013; 8: 218-224

$103 \mathrm{Yu}$ Z, Han S, Zhu J et al. Pre-pregnancy body mass index in relation to infant birth weight and offspring overweight/obesity: a systematic review and meta-analysis. PLoS One 2013; 8: e61627

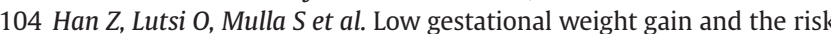
of preterm birth and low birthweight: a systematic review and metaanalyses. Acta Obstet Gyn Scan 2011; 90: 935-954

105 Rahman MM, Abe SK, Kanda $M$ et al. Maternal body mass index and risk of birth and maternal health outcome in low- and middle-income countries: a systematic review and meta-analysis. Obes Rev 2015; 16 : 758-770

106 Alcazar MAA, Nüsken E, Nüsken KD. Programmierung durch intrauterine Mangelversorgung. Monatsschr Kinderheilkd 2016; 164: 106113

107 Gaillard R. Maternal obesity during pregnancy and cardiovascular development and disease in the offspring. Eur J Epidemiol 2015; 30: $1141-1152$

108 van Lieshout RJ. Role of maternal adiposity prior to and during pregnancy in cognitive and psychiatric problems in offspring. Nutr Rev 2013; 71: S95-S101

109 van Lieshout RJ, Taylor VH, Boyle MH. Pre-pregnancy and pregnancy obesity and neurodevelopmental outcomes in offspring: a systematic review. Obes Rev 2011; 12: e548-e559

110 Blumfield ML, Hure AJ, Macdonald-Wicks L et al. Systematic review and meta-analysis of energy and macronutrient intakes during pregnancy in developed countries. Nutr Rev 2012; 70: 322-336

111 Blumfield ML, Hure AJ, Macdonald-Wicks L et al. Micronutrient intakes during pregnancy in developed countries: systematic review and meta-analysis. Nutr Rev 2013; 71: 118-132

112 Muff C, Weyers S. Sozialer Status und Ernährungsqualität. Evidenz, Ursachen und Interventionen. Ernährungsumschau 2010; 10: 84-89

113 Beijers R, Buitelaar JK, de Weerth C. Mechanisms underlying the effects of prenatal psychosocial stress on child outcomes: beyond the HPA axis. Eur Child Adolesc Psychiatry 2014; 23: 943-956

114 Entringer S, Buss C, Wadhwa PD. Prenatal stress, development, health and disease risk: A psychobiological perspective-2015 Curt Richter Award Paper. Psychoneuroendocrinology 2015; 62: 366-375

115 Alder J, Fink N, Bitzer J. Depression and anxiety during pregnancy: a risk factor for obstetric, fetal and neonatal outcome? A critical review of the literature. J Matern Fetal Neonatal Med 2007; 20: 189-209
116 Grigoriadis S, VonderPorten EH, Mamisashvili L et al. The impact of maternal depression during pregnancy on perinatal outcomes: a systematic review and meta-analysis. J Clin Psychiatry 2013; 74: e321e341

$117 \mathrm{Hu}$ R, Li Y, Zhang Z et al. Antenatal depressive symptoms and the risk of preeclampsia or operative deliveries: a meta-analysis. PLoS One 2015; 10: e0119018

118 Bussières EL, Tarabulsy GM, Pearson J et al. Maternal prenatal stress and infant birth weight and gestational age: a meta-analysis of prospective studies. Dev Rev 2015; 36: 179-199

119 Ding $X X, W u Y L, X u$ SJ et al. Maternal anxiety during pregnancy and adverse birth outcomes: a systematic review and meta-analysis of prospective cohort studies. J Affect Disord 2014; 159: 103-110

120 Staneva A, Bogossian F, Pritchard $M$ et al. The effects of maternal depression, anxiety, and perceived stress during pregnancy on preterm birth: a systematic review. Women Birth 2015; 28: 179-193

121 Grote NK, Bridge JA, Gavin AR et al. A meta-analysis of depression during pregnancy and the risk of preterm birth, low birth weight, and intrauterine growth restriction. Arch Gen Psychiatry 2010; 67: 10121024

122 Szegda K, Markenson G, Bertone-Johnson ER et al. Depression during pregnancy: a risk factor for adverse neonatal outcomes? A critical review of the literature. J Matern Fetal Neonatal Med 2014; 27: 960 967

123 Martini J, Knappe S, Garthus-Niegel S et al. Psychische Störungen in den reproduktiven Phasen der Frau: Häufigkeiten, Verlauf und Besonderheiten. Fortschr Neurol Psychiatr 2016; 84: 432-449

124 Paschetta E, Berrisford G, Coccia F et al. Perinatal psychiatric disorder: an overview. Am J Obstet Gynecol 2014; 210: 501-509

125 Goodman SH. Depression in mothers. Annu Rev Clin Psychol 2007; 3 107-135

126 Kaitz M, Maytal H. Interactions between anxious mothers and their infants: an integration of theory and research findings. Infant Ment Health 2005; 26: 570-597

127 Stewart RC. Maternal depression and infant growth: a review of recent evidence. Matern Child Nutr 2007; 3: 94-107

128 Graignic-Philippe R, Dayan J, Chokron S et al. Effects on prenatal stress on fetal and child development: a critical review. Neurosci Biobehav R 2014: 43: 137-162

129 Stein A, Pearson RM, Goodman SH et al. Effects of perinatal mental disorders on the fetus and child. Lancet 2014; 384: 1800-1819

130 Kingston D, McDonald S, Austin MP et al. Association between prenatal and postnatal psychological distress and toddler cognitive development: a systematic review. PLoS One 2015; 10: e0126929

131 Kingston D, Tough S, Whitfield H. Prenatal and postpartum maternal psychological distress and infant development: a systematic review. Child Psychiat Hum D 2012; 43: 683-714

132 Tarabulsy GM, Pearson J, Vaillancourt-Morel MP et al. Meta-analytic findings of the relation between maternal prenatal stress and anxiety and child cognitive outcome. J Dev Behav Pediatr 2014; 35: 38-43

133 Waters CS, Hay DF, Simmonds JR et al. Antenatal depression and children's developmental outcomes: potential mechanisms and treatment options. Eur Child Adolesc Psychiatry 2014; 23: 957-971

134 Woods SM, Melville JL, Guo YQ et al. Psychosocial stress during pregnancy. Am J Obstet Gynecol 2010; 202: 61.e1-61.e7

135 Gavin NI, Gaynes BN, Lohr KN et al. Perinatal depression: a systematic review of prevalence and incidence. Obstet Gynecol 2005; 106: 10711083

136 Goodman JH, Chenausky KL, Freeman MP. Anxiety disorder during pregnancy: a systematic review. J Clin Psychiatry 2014; 75: e1153e1184

137 Martini J, Asselmann E, Einsle F et al. A prospective-longitudinal study on the association of anxiety disorders prior to pregnancy and pregnancy- and child-related fears. J Anxiety Disord 2016; 40: 58-66

138 Liepe K, Blättner B. Gewalterfahrungen in der Schwangerschaft: Prävalenzstudien in den OECD-Ländern. Gesundheitswesen 2013; 75 : 473-480

139 Chambliss LR. Intimate partner violence and its implication for pregnancy. Clin Obstet Gynecol 2008; 51: 385-397

140 De Almeida CP, Sá E, Cunha F. Violence during pregnancy and its effects on mother-baby relationship during pregnancy. J Reprod Infant Psyc 2013; 31: 370-380

141 Chan KL, Brownridge DA, Fong DYT et al. Violence against pregnant women can increase the risk of child abuse: a longitudinal study. Child Abuse Negl 2012; 36: 275-284 
142 Dixon L, Browne K, Hamilton-Giachritsis C. Risk factors of parents abused as children: a mediational analysis of the intergenerational continuity of child maltreatment (part I). J Child Psychol Psychiatry 2005; 46: 47-57

143 Kersting A. Peripartale Depressionen und Trauer nach Schwangerschaftsverlusten. Besondere Problemfelder in der Geburtshilfe. Nervenarzt 2012; 83: 1434-1441

144 McDonald K, Amir LH, Davey MA. Maternal bodies and medicines: a commentary on risk and decision-making of pregnant and breastfeeding women and health professionals. BMC Public Health 2011; 11: S5
145 Crozier SR, Robinson SM, Borland SE et al. Do women change their health behaviours in pregnancy? Findings from the Southampton Women's Survey. Paediatr Perinat Epidemiol 2009; 23: 446-453

146 Deutsche Hauptstelle für Suchtfragen (DHS), Hrsg. Factsheet: Alkohol in der Schwangerschaft. März 2010. Online: http://www.dhs.de/ fileadmin/user_upload/pdf/Factsheets/100319_Factsheet_FASD_-_ DIN.pdf; last access: 15.08 .2016

147 Stiegler A, Bieber L, Karacay K et al. Barrieren in der Thematisierung des Tabak- und Alkoholkonsums Schwangerer in der gynäkologischen Praxis. Eine Fokusgruppenstudie mit Frauenärzten. Gesundheitswesen 2015; DOI: 10.1055/s-0035-1548935

148 Schild RL. Soziale Vorsorge und allgemeine Beratung in der Schwangerschaft. Gynäkologe 2015; 48: 736-740 